\title{
Establishing the effectiveness of technology-enabled dementia education for health and social care practitioners: a systematic review
}

Kevin Muirhead ${ }^{1 *} \mathbb{D}$, Leah Macaden ${ }^{1}$, Keith Smyth², Colin Chandler ${ }^{3}$, Charlotte Clarke $^{4}$, Rob Polson ${ }^{5}$ and Chris O'Malley ${ }^{5}$

\begin{abstract}
Background: Dementia prevalence is increasing globally and yet evidence suggest that gaps exist in dementiaspecific knowledge among health and social care practitioners. Technological modes of educational delivery may be as effective as traditional education and can provide practitioners with increased accessibility to dementia training. Benefits of digitally based dementia education have been established including pedagogical strategies that influence dementia knowledge and care attitudes. This review aimed to appraise and synthesise contemporary experimental evidence that evaluated technology-enabled dementia education for health and social care practitioners. Outcomes based on Kirkpatrick's Model were learner satisfaction; knowledge, skills, and attitudes; behaviours; and results.

Methods: MEDLINE, CINAHL, and Web of Science were among 8 bibliographic databases searched from January 2005 until February 2020. Keywords included dementia and education (and terms for technological modes of education, learning, or training). We included experimental and quasi-experimental studies. Medical Education Research Study Quality Instrument established the overall quality of included studies and pragmatic application of Mixed Methods Appraisal Tool established individual study quality and highlighted methodological features of educational research. Narrative synthesis was conducted as heterogeneous outcome data precluded meta-analysis.

Results: We identified 21 relevant studies: 16 evaluated online dementia education and 5 evaluated computer-based approaches. Most studies used before-after designs and study quality was moderate overall. Most studies reported knowledge-based outcomes with statistically significant findings favouring the training interventions. Positive effects were also observed in studies measuring skills and attitudinal change. Fewer studies reported significant findings for behavioural change and results due to training. Case-based instruction was a frequently described instructional strategy in online dementia education and videos were common information delivery modes. CD-ROM training and simulation activities were described in computer-based dementia education.
\end{abstract}

Discussion: Future emphasis must be placed on teaching and learning methods within technology-enabled dementia education which should be role relevant and incorporate active and interactive learning strategies. Future

\footnotetext{
*Correspondence: 17028759@uhi.ac.uk

1 Department of Nursing \& Midwifery, School of Health, Social Care \&

Life Sciences, University of the Highlands and Islands, Centre for Health

Science, Old Perth Road, Inverness IV2 3JH, UK

Full list of author information is available at the end of the article
} original author(s) and the source, provide a link to the Creative Commons licence, and indicate if changes were made. The images or other third party material in this article are included in the article's Creative Commons licence, unless indicated otherwise in a credit line to the material. If material is not included in the article's Creative Commons licence and your intended use is not permitted by statutory regulation or exceeds the permitted use, you will need to obtain permission directly from the copyright holder. To view a copy of this licence, visit http://creativecommons.org/licenses/by/4.0/. The Creative Commons Public Domain Dedication waiver (http://creativeco mmons.org/publicdomain/zero/1.0/) applies to the data made available in this article, unless otherwise stated in a credit line to the data. 
evaluations will require contextually relevant research methodologies with capacity to address challenges presented by these complex educational programmes and multi-component characteristics.

Systematic review registration: This systematic review is based on a protocol registered with PROSPERO (CRD42 018115378).

Keywords: Dementia, Dementia education, Dementia training, Technology-enabled learning, Systematic review, Effectiveness

\section{Background}

Dementia is a global public health priority and significant challenge for health and social care [1,2]. Fifty million people are estimated to be living with dementia globally and the prevalence is anticipated to rise to 152 million by 2050 [3]. Within the UK, 850,000 people (1 in 14 adults over the age of 65) are estimated to be living with dementia and future prevalence is predicted to mirror global trends [4]. In Scotland, dementia has been a national priority since 2007 [5], prompting a series of national strategies for better dementia care and services [6-8]. Concern about the quality of care for people living with dementia has intensified the need for an appropriately educated workforce [9] with evidence suggesting gaps in dementiaspecific knowledge among practitioners [10].

The dementia care setting is comprised of health and social care services that are delivered within hospitals, primary care, residential, and nursing homes, as well as community care [11]. Dementia education is required in all care contexts. Skilled, knowledgeable, and dementia competent staff are critical for person-centred dementia care in the acute hospital [12]. Hospital environments can be unsuitable for dementia care and inadequate staff training can result in unmet care needs and an increase in behavioural and non-cognitive symptoms of dementia-which staff report to be burdensome [13]. In primary care, dementia education is particularly helpful to support early diagnosis, appropriate treatment, and on-going support $[14,15]$. However, the primary care workforce has had limited access to dementia education and a range of training needs have been identified [16]. Inadequate caregiver training in social care environments including care homes can result in low-level staff morale and staff retention difficulties that negatively impact care quality [17]. Furthermore, it is essential that the early clinical experiences of health and social care practitioners (HSCPs) is underpinned by clear and relevant undergraduate dementia education that is related to the knowledge, skills, and attitudes that are required to care effectively for people living with dementia [18].

Technology-enabled dementia education (TEDE) may provide learning opportunities for HSCPs through increased accessibility to dementia training in the multiple practice contexts. TEDE refers to a collection of methods that use the application of some form of digital technology for teaching and/or learning in the dementia education context. The word enabled refers to facilitation: dementia education is made possible by the use of technology (definition adapted [19]). TEDE programmes encompass several subsets of delivery methods not limited to e-learning, online learning, and other computerbased learning modalities including blended methods that integrate face-to-face teaching and learning with technological approaches. These delivery methods have their own nuance of meaning and subset relationships exist between them (Fig. 1). The concept of internetbased learning, for example, is broader than web-based learning since the web is only one of many internet services. Online learning can be arranged through many networks, so, internet-based learning is only a subset of online learning. Computer-based learning implies that the computer is not connected to a network; therefore, computer-based learning is not a subset of online learning. E-learning can take place via any electronic medium, so, online learning and computer-based (non-networked) learning are both subsets of e-learning [20]. Definitions for the key terms are shown in Table 1. The definitions highlight that subset terms may be, and often are, used interchangeably which is a potential source of confusion. Furthermore, the pedagogical aspect of the term (e.g., learning) may be replaced with synonyms such as education/instruction/training. In the TEDE context, the pedagogical aspect may be specified further (e.g., dementia education).

E-learning has gained popularity due to cost effectiveness, high flexibility, and reduced dependence on geographical boundaries [22]. Evidence suggests that it is as effective, and possibly superior to traditional learning for undergraduate health professionals [23]. Among licensed healthcare practitioners, e-learning is associated with no important benefits compared to traditional learning [22]; however, the relative efficacy of e-learning must also factor critical dimensions relating to accessibility and acceptability. In a review of dementia training programmes for staff working in general hospital settings, Scerri et al. [24] reported on a study where the uptake of a self-directed dementia related e-learning programme among nurses was poor. 


\section{Technology-enabled approaches to Dementia Education}

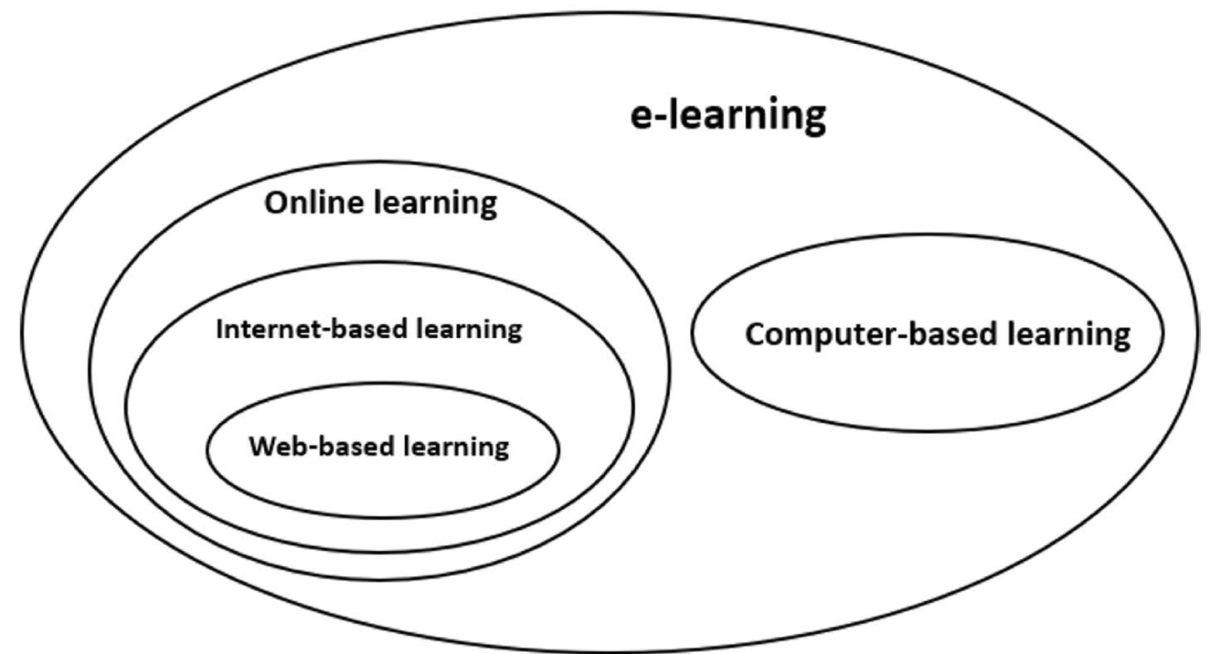

Fig. 1 Relationship of key terms in technology-enabled dementia education. Adapted [20]

Table 1 Definitions of key terms in technology-enabled dementia education

\begin{tabular}{ll}
\hline Term & Definition \\
\hline TEDE & $\begin{array}{l}\text { A collection of methods that use the application of some form of digital technology for teaching and/or learning in the } \\
\text { dementia education context. } \\
\text { Learning via any electronic medium. E-learning refers to the application of information communication technology in its } \\
\text { widest sense to support and improve the learning experience. Online and non-networked computer-based learning are } \\
\text { subsets of e-learning. } \\
\text { Online learning refers to any e-learning that is conducted online. Internet-based learning and web-based learning are } \\
\text { subsets of online learning. The terms are often used interchangeably. }\end{array}$ \\
Online learning & $\begin{array}{l}\text { This is a non-networked approach for e-learning that emphasises the use of a computer (or computerised device) as the } \\
\text { delivery platform. } \\
\text { A mixed mode of delivery combining face-to-face learning with e-learning techniques. It is especially relevant to intro- } \\
\text { Blended learning }\end{array}$
\end{tabular}

The definitions were formulated for the review context and adapted from relevant sources [19-21]

TEDE Technology-enabled dementia education

The review suggested that e-learning may not be feasible in the acute hospital setting due to limitations in participants' time, internet access, and digital competence. Surr and Gates [25] reported on the same study where 26 staff signed up to undertake the training but only six people completed it. Indeed, all participating staff chose to complete the education modules in hard copy rather than online [26]. Furthermore, Surr et al. [27] reported completion rates from a study of online dementia education being only $50 \%$. These findings are not consistent across all reviews of dementia education. For instance, Elliot et al. [11] reported that satisfaction and compliance to attend dementia training was higher among nurses who completed training using a computer resource compared to nurses who attended traditional group training in a lecture format.

There is evidence to suggest that the flexibility of e-learning can be beneficial [27]; however, Surr and Gates [25] highlighted that approaches that rely on individuals to schedule their own time for dementia training, including e-learning, may lead to poorer outcomes in terms of knowledge gains and attitudinal change. The evidence that e-learning can contribute directly to these learning outcomes is mixed and may be dependent upon more nuanced pedagogical methods. Surr et al. [27] reported that confidence, competence, and self-efficacy were achieved following interactive web-based resources with evidence suggesting that non-interactive approaches may 
be less effective. In general, active learning, for example, using online multimedia was considered to be more effective than passive approaches such as watching an online video lecture. Where e-learning was utilized, learners preferred a combination of individual study with opportunities for online or face-to-face discussion. Online discussions were felt to be particularly beneficial to learning; however, time demands for learners and facilitators and the need for specialist technical support suggested this as a resource intensive form of study [27].

Scerbe et al. [28] conducted a review that focused exclusively on digital tools for the delivery of dementia education for health-care providers. The review included 10 studies that used pre- and post-test measures of evaluation. The teaching and learning methods detailed within studies included videos, audio-narration, graphics, and some interactive content including discussion forums. The review established that all of the included studies demonstrated a positive change on the outcomes measured and the review concluded there was compelling confirmation of effectiveness for digitally conveyed dementia education. Scerri et al. [24] highlighted that the heterogeneity of dementia training programmes can make it difficult to determine whether outcomes can be attributed to the interventions. Therefore, methods to evaluate TEDE may require capacity to demonstrate outcomes based on specific delivery methods and also the more nuanced teaching and learning methods contained within training programmes.

The pace of technological progress also requires consideration as this may influence pedagogical practices and subsequent learning outcomes. Web 2.0, for instance, resulted in a paradigm shift for teaching and learning online. Adopted in popular commentary in 2005 [29], Web 2.0 describes the transformation of the static 'read only' Web 1.0 into a dynamic 'read-and-write' participatory media [30]. This has facilitated interconnectivity and the interactive learning opportunities which are likely to be valuable when using technology for dementia education [27]. Therefore, contemporary TEDE programmes may have additional capacity to harness the interactive strategies including peer and instructor supported collaboration and discussion. However, the internet services required to enable this type of interactivity may not be universally available, particularly in rural communities with poor technological infrastructure and limitations in broadband and mobile internet coverage [31]. Therefore, at present, non-networked computer-based approaches may continue to play an important role in dementia education for those practitioners who are less digitally included.

Much of the current evidence on TEDE is from practice-based settings using study designs involving pre- and post-tests [28]. Experimental methods in educational research are, however, diverse [32] and there is potential for more to be known about the role of quantitative methods that evaluate TEDE programmes. For instance, studies involving comparator groups may provide insight into the relative value of different training approaches. Furthermore, studies from higher educational settings may provide valuable sources of additional information including innovative pedagogical practices. The complexities of a review of TEDE for HSCPs will benefit by incorporating a robust framework for data synthesis and presentation of the review findings. Previous reviews of dementia education have applied Kirkpatrick's four-level model [24, 25, 27]; a widely cited framework for evaluating educational and training interventions. Each level denotes a particular value added from training investment including learners' reactions to the training; learning gains as knowledge, skills, and attitudinal change; practice-based behaviour changes following training; and the wider results due to the training [33].

\section{Aim}

The aim of this review was to systematically appraise and synthesise the current experimental evidence evaluating technology-enabled dementia education programmes for health and social care practitioners. The research questions were:

- What are the experimental research methods that evaluate technology-enabled dementia education programmes for health and social care practitioners?

- What are the methodological strengths and limitations of experimental studies that evaluate technology-enabled dementia education programmes for health and social care practitioners?

- Are online and non-networked computer-based dementia education programmes beneficial across the outcomes in Kirkpatrick's model?

- What are the delivery methods, instructional strategies, and modes of information delivery in technology-enabled dementia education programmes for health and social care practitioners?

- What instructional strategies support interactivity (communication and collaboration) in technologyenabled dementia education for health and social care practitioners?

\section{Methods}

The design and methodology of this systematic review were informed using the Preferred Reporting Items for Systematic Review and Meta-Analysis (PRISMA) 
guidelines and checklist [34]. The checklist is included (Additional file 1).

\section{Protocol}

A protocol was registered on PROSPERO (CRD42018115378) and published to describe a systematic mixed methods review detailing the characteristics and effectiveness of TEDE [35]. The review has been completed and submitted for publication. The current review expands on the quantitative evidence and methods to evaluate the effectiveness of TEDE for HSCPs.

\section{Criteria for considering studies for this review Eligibility criteria}

We included studies involving HSCPs with, without, or working toward a professional qualification or registration participating in TEDE in a workplace or educational setting. We did not include studies of TEDE for informal/family caregivers or people living with dementia. We included all TEDE courses, modules, and standalone resources delivered in online, computerbased, and blended learning programmes. Decision support, DVD/video, and telephonic interventions were not included. We included experimental and observational study designs that involved the systematic collection of data and comparison of intervention effects in the evaluation of TEDE programmes. This included study designs that involved one group or more than one group of participants. Study designs involving one group of participants included before-after designs; interrupted time-series designs; and repeated measure designs. Study designs involving more than one group of participants included randomised trials and nonrandomised studies-including controlled before-after and time-series designs [36]. These eligible study designs were categorised into two groups: experimental and quasi-experimental studies. Studies were judged to be experimental where the investigator randomly allocated participants to a treatment (TEDE) and a control/comparator group. Studies were considered to be quasi-experiments where they lacked the key feature of experimental studies-randomisation. Quasi-experimental studies included those with a control/comparator group (e.g., non-randomised studies) and those without a control/comparator group (e.g., before-after studies) [37]. Non-randomised studies also included quasi-randomised studies where methods of allocation were known but were not strictly random. Quasiexperimental studies shared with experimental studies a similar purpose-to test causal hypotheses. In experimental studies, random allocation creates two or more groups that are probabilistically similar to one another; therefore, any difference between the groups is likely to be due to the 'treatment'. This feature of experimental research is highly valued and randomised trials are considered to be the 'gold standard' in research [38]. Quasiexperimental studies can also aid in understanding causal effects; however, the reliability of causal claims and estimates varies across these designs and depends on how close the study conditions are to an experiment [39]. Therefore, we considered the eligible study designs in a relative hierarchy, in terms of establishing causality, and provided definitions with relevance to the review context (Table 2). We included the experimental and quasi-experimental evidence from quantitative and mixed method evaluations of TEDE. We did not include qualitative studies or studies that only evaluated TEDE programmes using descriptive narrative or

Table 2 Experimental and quasi-experimental study designs

\begin{tabular}{|c|c|c|c|}
\hline Classification & Category & Study design & Definition \\
\hline Experimental & 1 & Randomised trials & $\begin{array}{l}\text { An experimental study in which individuals or groups are allocated to different } \\
\text { interventions using methods that are random. }\end{array}$ \\
\hline \multirow[t]{5}{*}{ Quasi-experimental } & 2 & Interrupted time-series designs & $\begin{array}{l}\text { A study that uses observation at multiple (at least three) time points before and after } \\
\text { an intervention. }\end{array}$ \\
\hline & 3 & Controlled before-after studies & $\begin{array}{l}\text { A study in which observations are made before and after the implementation of an } \\
\text { intervention, both in a group that receives the intervention and in a control group } \\
\text { that does not. }\end{array}$ \\
\hline & 4 & Non-randomised studies & $\begin{array}{l}\text { A study in which people are allocated to different interventions using methods that } \\
\text { are not random. }\end{array}$ \\
\hline & 5 & Before-after studies & $\begin{array}{l}\text { A study in which observations are made before and after the implementation of an } \\
\text { intervention in the same group of individuals. }\end{array}$ \\
\hline & 5 & Repeated measure studies & $\begin{array}{l}\text { A before-after study in which there are multiple post-intervention time points at } \\
\text { which outcome measurements are made [40]. }\end{array}$ \\
\hline
\end{tabular}

Category 1 studies are considered to be the most robust, and category 5 studies the least robust, in terms of establishing causality. Hierarchies of quasi-experimental studies informed by Harris et al. [41]. Definitions of study designs were adapted from Cochrane Effective Practice and Organisation of Care (EPOC) guidance [36] unless otherwise indicated 
survey data of participants' general impressions. Studies published before 2005 were excluded to reflect the pace of digital change and technological progress since Web 2.0 [42] . Studies not published in the English language were excluded as resources for translation were not available.

\section{Outcome measures}

The primary outcome measures were based on Kirkpatrick's four-level model which is the most renowned and widely used evaluation model for educational and training interventions $[43,44]$. The model was adapted for the review context to identify relevant outcomes from primary studies and to provide a framework for data synthesis and presentation of the review findings [45]. We adapted the model for greater emphasis and delineation of the level 2 sub-items (knowledge, skills, and attitudes) as each, in isolation, was considered to be an important learning outcome in the dementia education context. Definitions for each level in the adapted model are provided in Table 3.

\section{Search methods}

Literature searches were carried out in MEDLINE (OVID interface), CINAHL Complete (EBSCO interface), ERIC (EBSCO interface), PsycINFO (EBSCO interface), PubMed, Web of Science Core Collection, OVID Nursing Database, and SCOPUS from January 2005 until November 2018. The search was updated in February 2020. MEDLINE and PubMed were both included to ensure that the search was comprehensive. MEDLINE is a subset of PubMed with the latter containing more material. Subject librarians (RP and COM) from the University of the Highlands and Islands were consulted in the development of the search strategy. Experts in TEDE were not contacted for other sources of information. The multi-database search strings are available (Additional file 2).

\section{Data collection and analysis Selection of studies}

The results from the literature search were stored in RefWorks research management software where duplicate citations were identified, confirmed, and removed. One reviewer (KM) then screened the titles and abstracts of the remaining studies. Two other reviewers (LM and CC) then screened $10 \%$ of the titles and abstracts-by each screening 5\%. Eligibility conflicts were resolved through discussion and third-party arbitration was not required. Full-text versions of potentially eligible studies were assessed for eligibility by one reviewer (KM). Ineligible studies were issued with an exclusion rationale and removed. Reference lists of eligible studies were screened by $\mathrm{KM}$ and any studies meeting the eligibility criteria were included. One reviewer (KM) examined the eligible study reports (papers) to establish any instances where more than one paper reported on the same training programme. Papers reporting duplicate evaluations were not eligible; however, any papers that reported on different aspects (i.e., multiple evaluations) of the same training programme were eligible for inclusion.

\section{Data extraction}

Data was extracted using a data extraction form designed specifically for the review. The form was pilot tested before application and study data was extracted by one reviewer (KM). A sample data extraction form is provided (Additional file 3).

\section{Assessment of methodological quality}

The Mixed Methods Appraisal Tool (MMAT) [46] and Medical Education Research Study Quality Instrument (MERSQI) [47] were used in a two-stage process for quality appraisal. MMAT is a generic critical appraisal

Table 3 Definitions for the levels of outcome evaluation

\begin{tabular}{lll}
\hline Level & Outcome & Definition \\
\hline 1 & Reaction & $\begin{array}{l}\text { This is a measure of how participants feel about aspects of the TEDE programme. It is a measure of learner } \\
\text { satisfaction. }\end{array}$ \\
$2 \mathrm{a}$ & Learning-change in knowledge & This is a measure of knowledge acquired as a result of the TEDE programme. \\
$2 \mathrm{~b}$ & Learning-change in skills & This is a measure of the skills acquired as a result of the TEDE programme. \\
$2 \mathrm{c}$ & Learning-change in attitudes & This is a measure of attitudinal change as the result of the TEDE programme. \\
3 & Behaviours & This is a measure of the extent to which participants change their behaviours in practice because of the TEDE \\
& Results & programme. \\
& This is a measure of results that occurred because of the TEDE programme. It includes outcomes for service \\
& users and other organisational-level outcomes.
\end{tabular}


tool with specific categories for qualitative research, randomised trials, non-randomised studies, quantitative descriptive studies, and mixed methods studies. The provision of multiple methodological quality criteria for different study designs makes it most relevant for quality appraisal in mixed studies reviews [48]. MMAT was considered to be appropriate for use in this non-mixed studies review as the tool was being used simultaneously by the study authors in a mixed methods review of TEDE. This was a pragmatic decision to achieve consistency between the two reviews given that MMAT has sufficient capacity to appraise the experimental design methodologies included in the current review. Each MMAT category has a specific criteria with three response options: 'yes' means that the criterion is met, 'no' means that the criterion is not met, and 'can't tell' means that there is not enough information in the paper to judge if the criterion is met. MERSQI puts emphasis on methodological rigour associated with experimental and quasi-experimental studies in medical education [49]. It is a reliable tool for appraising the quality of medical education research and has been used previously to complement other tools [50]. MERSQI has 6 domains: study design, sampling, type of data, validity of evaluation instrument, data analysis, and outcomes. Each domain includes items which are scored based on the methodological strengths of primary studies. The maximum domain score is 3 and the maximum total MERSQI score is 18. MMAT was used to assess the quality of individual studies and MERSQI established the overall quality of TEDE research. The combined approach to quality appraisal also aimed to highlight unique methodological features of educational research for consideration in the context of generic appraisal. Two reviewers (KM and LM or KS) appraised 19\% of studies using MMAT. Disagreements were resolved through discussion. All other quality appraisal was conducted by one reviewer (KM).

\section{Data synthesis}

All of the studies included in the review reported findings quantitatively; however, it was not appropriate to undertake meta-analyses due to the heterogeneity of study designs and statistical data. Instead, findings from the primary studies were summarised in a narrative synthesis using textual description and tabulation. A systematic approach was applied to the synthesis. Firstly, studies of online dementia education (ODE) and non-networked computer-based dementia education (CBDE) were identified and synthesised separately. For each e-learning approach, pedagogical strategies were discussed and key study information including intervention characteristics were presented in a summary table. Kirkpatrick's model provided a framework for subsequent synthesis where shared outcomes between studies were grouped together. Study designs and outcome measures within studies were then discussed before further delineation of findings according to the study setting (i.e., practice or higher education). Study findings were discussed with a particular focus on those studies that involved comparator/ control groups-allowing inference to optimal training approaches. Finally, key study findings were tabulated. The effects of interventions were reported at the level of each individual study. Each study was classified to show if the TEDE programme resulted in evidence of an effect, no evidence of an effect, or partial evidence of an effect (where a study reported inconsistent effects due to multiple outcome measures) for the outcomes measured. Effects were based on statistically significant pre- to posttest increases or between group differences that favoured treatment (TEDE) groups. Analysis of sub-groups was not undertaken; however, studies that included follow-up data were identified for inference on the sustainability of training effects over time.

\section{Results \\ Description of studies \\ Results of the search}

A total of 935 records were identified. Duplicate records (453) were removed and the titles and abstracts of 482 remaining records were screened based on the eligibility criteria. From these, 417 records were considered to be ineligible and the full texts of 65 records were retained for full-text review. Forty-five records were excluded as they focused on descriptive research using narrative or survey-based evaluations of TEDE programmes; did not include TEDE programmes; were not relevant to the review outcomes; or included non-HSCPs (family carers). The remaining 20 studies were included in the final synthesis with an additional study identified from the reference lists of eligible studies (Fig. 2).

\section{Included studies}

Key study and intervention characteristics from 21 eligible studies are demonstrated in Fig. 3. Full details of the included studies are provided in the Characteristics of Included Studies (Additional file 4).

\section{Excluded studies}

Studies considered ineligible for inclusion following fulltext review are available. Exclusion rationales are provided (Additional file 5).

\section{Study designs}

Four studies were randomised trials $[1,17,68,69]$ of which two were cluster randomised trials $[1,68]$. There were no interrupted time-series designs. Six studies were 


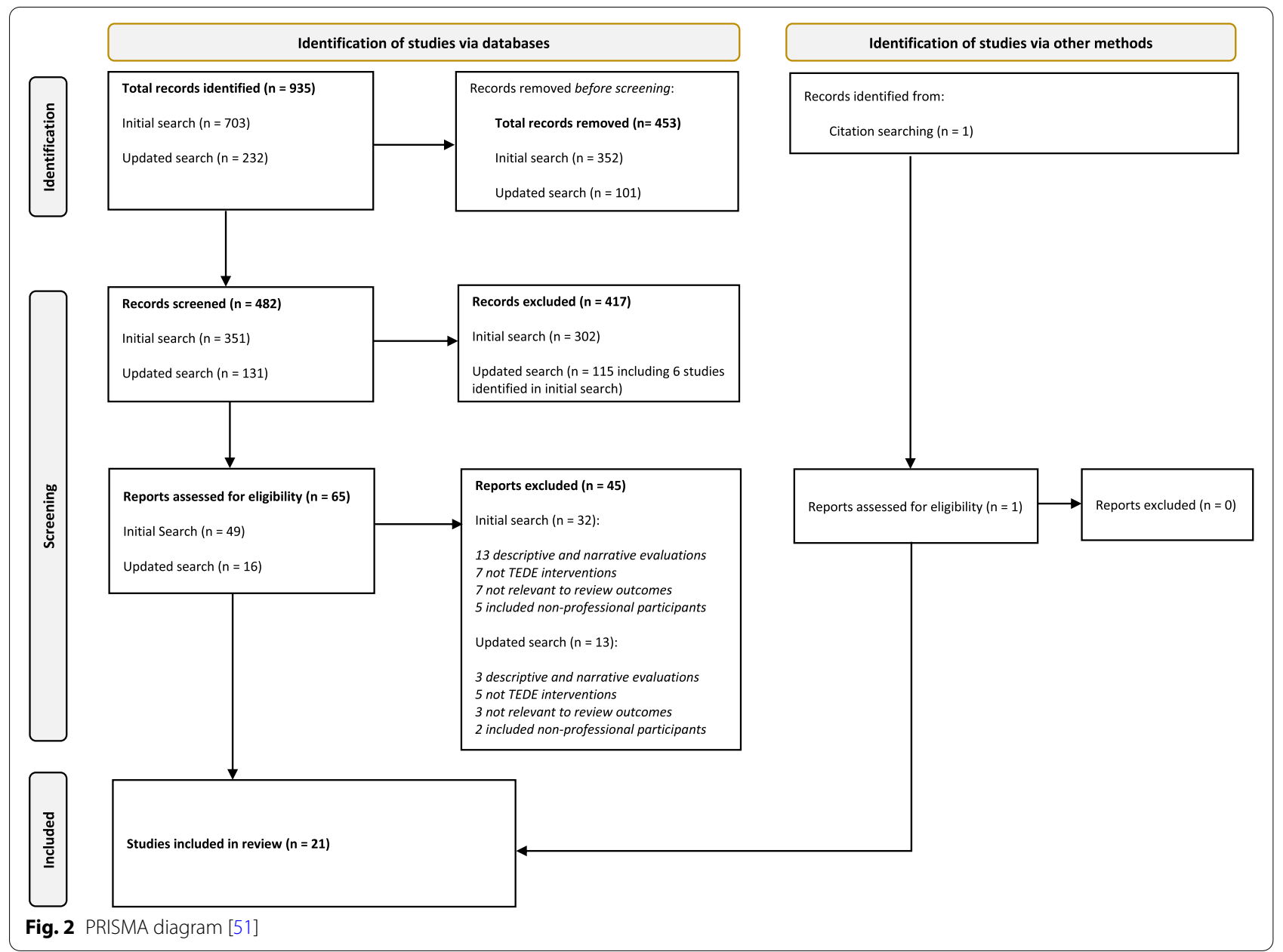

classified as being controlled before-after designs [53, 61, $63,64,66,67]$. Two of these studies described random allocation processes [64, 67]; however, they were not classified as randomised trials. One of the studies described random assignment to groups from a convenience sample [64] and the other introduced non-randomised participants into the experimental group [67]. There was one non-randomised study [55] and two studies used repeated measures [52, 62]. Eight before-after studies were included [14, 54, 56-60, 65] (Fig. 4). Six of the included studies were exploratory pilot studies [57-59, 62, 63, 67].

\section{Quality assessment of included studies MMAT}

Experimental studies (MMAT2) Randomisation processes were not described sufficiently in two of the trials $[17,69]$. Between group incomparability was identified in one trial [1], and it was not clear if between group similarities were significant in another [68]. An arbitrary threshold was applied for the assessment of outcome data. Acceptable dropout rates were considered to be $<20 \%$, which negatively affected judgments on the quality of three of the trials $[1,68,69]$. One trial was unblinded [1], and it was not possible to tell if outcome assessors were blinded in two trials $[68,69]$. Participant adherence may have been compromised in an unsupervised online dementia training [17]. Non-adherence was more apparent where intervention 'non-users' were identified [68], and where a trial ended prematurely due to participant dissatisfaction [69].

Quasi-experimental studies (MMAT3) There were a number of reporting limitations in quasi-experimental studies. It was frequently not possible to determine if participants were representative of target populations. Sampling methods were often not described, or there was insufficient information in relation to sampling or target populations. Convenience sampling was particularly problematic when assessing representativeness. It was frequently not possible to determine if outcome measures were appropriate. Limitations included inadequate 


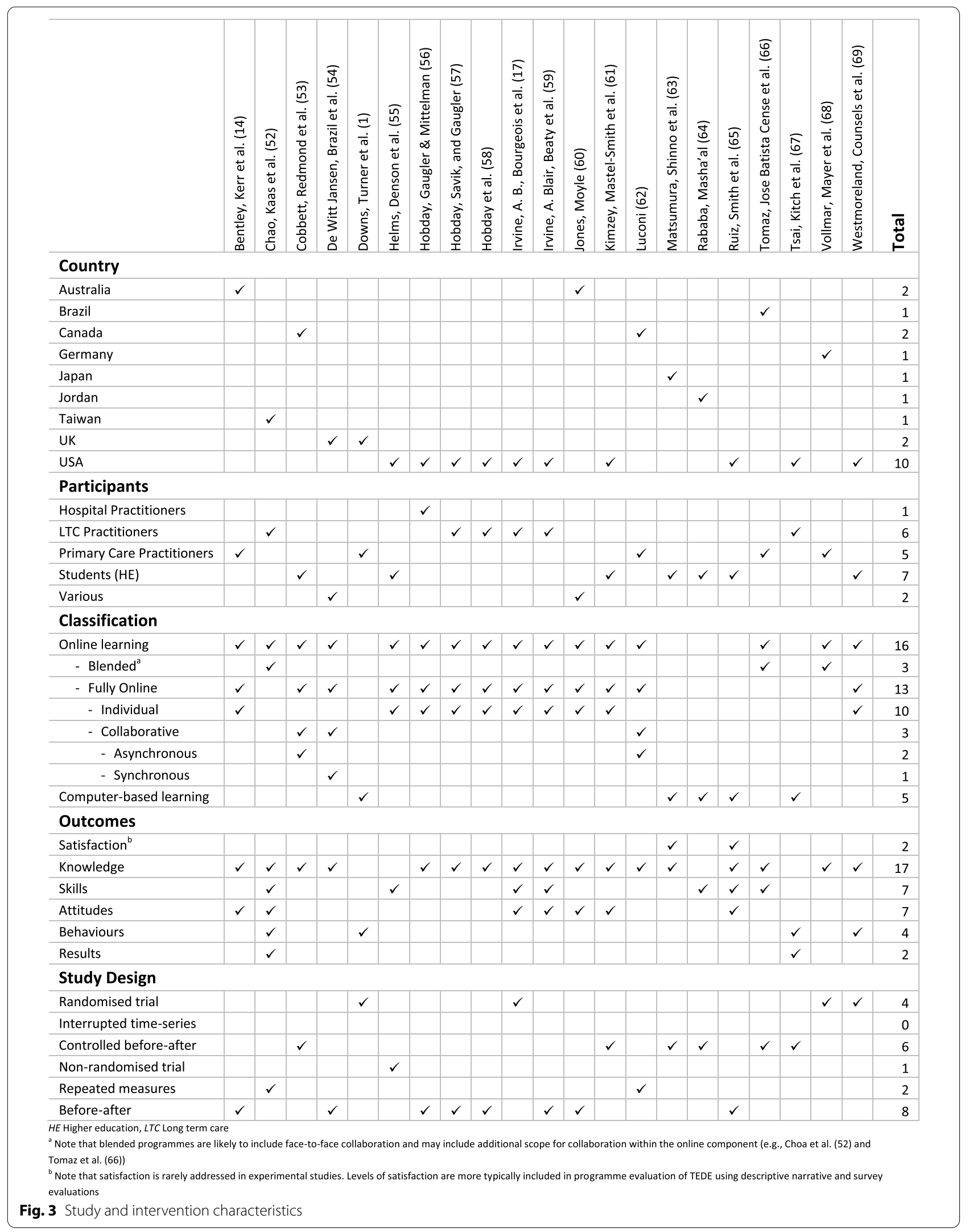




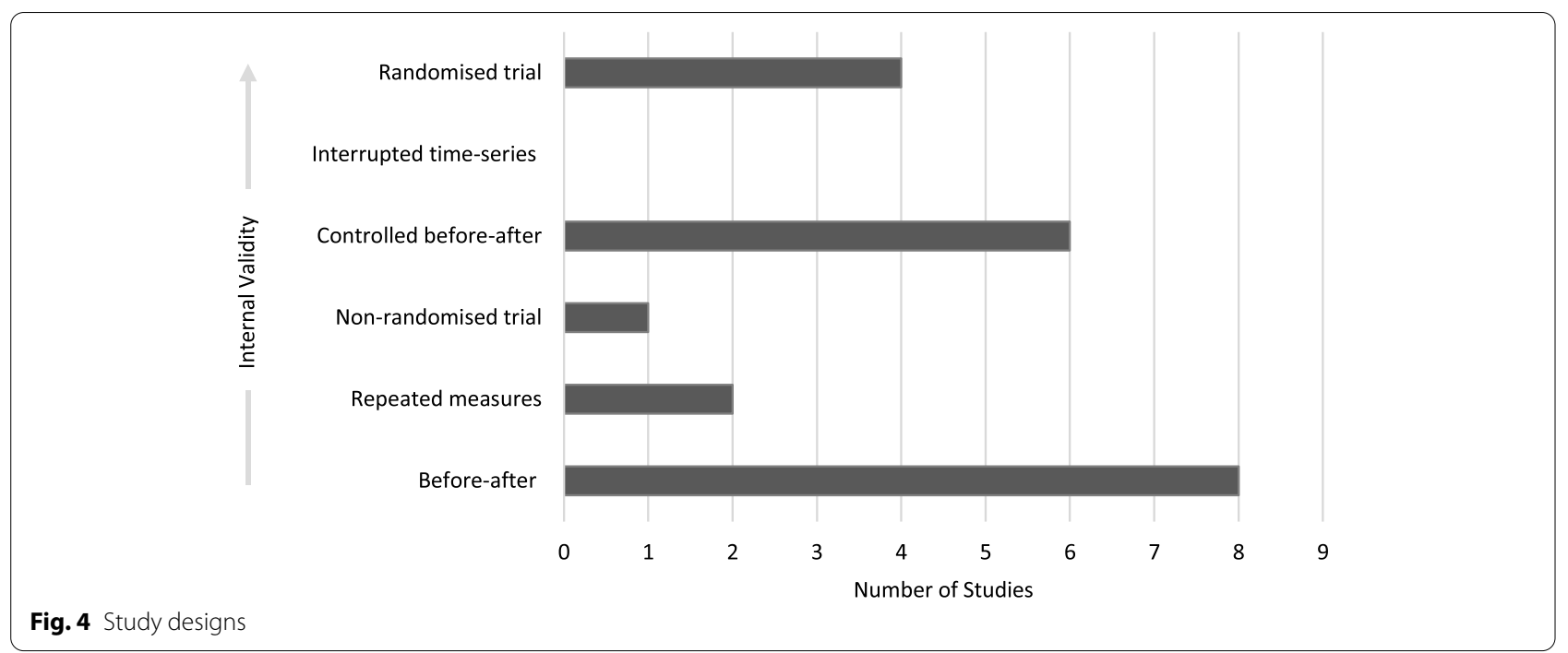

reports of validity or reliability (e.g., [55]); partial reporting of valid/reliable measures in studies using multiple measures (e.g., [60]); reports of validated measures that may not be reliable and vice versa (e.g., [56]); previously validated measures that were not validated in context (e.g., [14]); and measures with questionable reliability from sub-optimal alpha levels (e.g., [53]). An arbitrary threshold was applied to determine the completeness of outcome data. Acceptable dropout rates were considered to be $<20 \%$. It was frequently not possible to tell if the outcome data was complete. This was a common issue in before-after studies due to suboptimal reporting of participant numbers at either pre- or post-test. Two studies reported outcome data below the desired threshold [57, 67]. Interventions were considered to have been administered as intended unless studies reported evidence to the contrary. Cannot tell judgments were generally applied to studies that reported limitations to study processes (e.g., [52]); or where there were insufficient assurances of intervention controls including the location of participation (e.g., [55]). The main threat to study quality was from confounding factors which were frequently not described or accounted for in the study design or analysis. Time difference between pre- and post-tests in before-after studies was a common source of potential maturation effects.

A summary of MMAT quality appraisal is shown in Fig. 5. The ratings and support for judgment are provided in the Characteristics of Included Studies (Additional file 4).

\section{MERSQI}

The mean MERSQI scores from primary studies are presented with the standard deviation (SD). Scoring information is available for each domain and subdomain. The total MERSQI score was 12.38 (SD 1.6) which was interpreted to be 'moderate' quality overall (Table 4). Scoring data for individual primary studies and a decision-making tool developed for consistency across the review context are provided (Additional file 6).

\section{Establishing the effectiveness of TEDE}

From 21 studies identified in this review, 16 studies described ODE programmes [14, 17, 52-62, 66, 68, 69] and 5 involved non-networked CBDE approaches [1, 6365,67 .

\section{Online dementia education programmes}

Twelve studies of ODE were from practice-based settings $[14,17,52,54,56-60,62,66,68]$ and 4 were from higher education [53, 55, 61, 69]. Thirteen studies reported on fully online programmes $[14,17,53-62,69]$ and 3 included blended learning approaches $[52,66,68]$. Thereafter, inconsistent use of terminology (e.g., resources, programmes, courses, or modules) between studies made classification approaches more challenging. In general, online courses were either labelled as such [53], or involved a protracted duration $[62,66]$. Modules were generally described in terms of duration and frequency $[14,54,57-60,68]$. Frequently, duration and frequency were not specified $[17,52,55,56,61,69]$.

\section{Interactivity}

Two studies of blended learning stipulated additional scope for interactivity within the online components [52, 66]. One specified discussions in asynchronous virtual 
Table 4 Medical Education Research Study Quality Instrument: domain and total scores

\begin{tabular}{|c|c|c|}
\hline & Possible score & Mean (SD) \\
\hline Study design & 3 & $1.95(0.6)$ \\
\hline Single group cross-sectional or single group post-test only & 1 & \\
\hline Single group pre-test and post-test & 1.5 & \\
\hline Nonrandomised, 2 groups & 2 & \\
\hline Randomised controlled trial & 3 & \\
\hline \multicolumn{3}{|l|}{ Sampling } \\
\hline Institutions studied & 1.5 & $1.05(0.5)$ \\
\hline 1 & 0.5 & \\
\hline 2 & 1 & \\
\hline 3 & 1.5 & \\
\hline Response rate, \% & 1.5 & $1.31(0.4)$ \\
\hline Not applicable & 0 & \\
\hline$<50$ or not reported & 0.5 & \\
\hline $50-74$ & 1 & \\
\hline$>75$ & 1.5 & \\
\hline Type of data & 3 & $2.33(0.9)$ \\
\hline Assessment by participants & 1 & \\
\hline Objective measurement & 3 & \\
\hline \multicolumn{3}{|l|}{ Validity of evaluation instrument } \\
\hline Internal structure & 1 & $0.52(0.5)$ \\
\hline Not applicable & 0 & \\
\hline Not reported & 0 & \\
\hline Reported & 1 & \\
\hline Content & 1 & $0.52(0.5)$ \\
\hline Not applicable & 0 & \\
\hline Not reported & 0 & \\
\hline Reported & 1 & \\
\hline Relationships to other variables & 1 & $0.05(0.2)$ \\
\hline Not applicable & 0 & \\
\hline Not reported & 0 & \\
\hline Reported & 1 & \\
\hline \multicolumn{3}{|l|}{ Data analysis } \\
\hline Appropriateness of analysis & 1 & $1.00(0.0)$ \\
\hline Inappropriate for study design or type of data & 0 & \\
\hline Appropriate for study design and type of data & 1 & \\
\hline Complexity of analysis & 2 & $1.95(0.2)$ \\
\hline Descriptive analysis only & 1 & \\
\hline Beyond descriptive analysis & 2 & \\
\hline Outcomes & 3 & $1.69(0.4)$ \\
\hline Satisfaction, attitudes, perceptions, opinions, general facts & 1 & \\
\hline Knowledge, skills & 1.5 & \\
\hline Behaviours & 2 & \\
\hline Patient/healthcare outcome & 3 & \\
\hline Total & 18 & $12.38(1.6)$ \\
\hline
\end{tabular}

SD Standard deviation 
Table 5 Key characteristics of online dementia education programmes

\begin{tabular}{|c|c|c|c|c|}
\hline Study & Setting/participants & Programme & Duration & Programme characteristics \\
\hline Bentley, Kerr et al. 2019 [14] & $\begin{array}{l}\text { Primary care } \\
\text { International medical } \\
\text { graduates and practice } \\
\text { nurses }\end{array}$ & $\begin{array}{l}\text { Online interactive educa- } \\
\text { tional resource (recognising, } \\
\text { diagnosing, and manag- } \\
\text { ing dementia in general } \\
\text { practice) }\end{array}$ & $\begin{array}{l}4 \text { modules } \\
\text { Total duration: } 3 \mathrm{~h}\end{array}$ & $\begin{array}{l}\text { Delivery method: fully } \\
\text { online } \\
\text { Instructional strategy: NS } \\
\text { MID: video; external learning } \\
\text { resources } \\
\text { Interactivity: NS }\end{array}$ \\
\hline Chao, Kaas et al. 2016 [52] & $\begin{array}{l}\text { Long-term care } \\
\text { Nurses }\end{array}$ & $\begin{array}{l}\text { Advanced Innovative } \\
\text { Internet-Based Communica- } \\
\text { tion Education Program }\end{array}$ & $\begin{array}{l}4 \text { modules } \\
\text { Total duration NS }\end{array}$ & $\begin{array}{l}\text { Delivery method: blended } \\
\text { learning } \\
\text { Instructional strategy: } \\
\text { case-based } \\
\text { MID: video; face-to-face } \\
\text { Interactivity: face-to-face } \\
\text { discussion; online discus- } \\
\text { sion NOS; online reflective } \\
\text { journaling }\end{array}$ \\
\hline $\begin{array}{l}\text { Cobbett, Redmond et al. } \\
2016 \text { [53] }\end{array}$ & $\begin{array}{l}\text { Higher education } \\
\text { Nursing students }\end{array}$ & $\begin{array}{l}\text { Alzheimer's disease and } \\
\text { other dementias care } \\
\text { course: adapted online } \\
\text { course }\end{array}$ & $\begin{array}{l}8 \text { online modules and } 1 \\
\text { face-to-face } \\
\text { Module duration: } 1 \mathrm{~h} \text { (with } \\
\text { additional } 2 \text { h of prepara- } \\
\text { tory work) }\end{array}$ & $\begin{array}{l}\text { Delivery method: fully } \\
\text { online }^{a} \\
\text { Instructional strategy: } \\
\text { case-based } \\
\text { MID: video; text } \\
\text { Interactivity: online discus- } \\
\text { sion (asynchronous) }\end{array}$ \\
\hline $\begin{array}{l}\text { De Witt Jansen, Brazil et al. } \\
2018 \text { [54] }\end{array}$ & $\begin{array}{l}\text { Primary and Secondary care, } \\
\text { Nursing Home, and Hospice } \\
\text { Physicians and nurses }\end{array}$ & $\begin{array}{l}\text { Tele-mentoring to enhance } \\
\text { assessment and manage- } \\
\text { ment of pain in advanced } \\
\text { dementia (based on Project } \\
\text { ECHO model) }\end{array}$ & $\begin{array}{l}5 \text { sessions } \\
\text { Session duration: } 1 \text { h } 15 \text { min }\end{array}$ & $\begin{array}{l}\text { Delivery method: fully } \\
\text { online } \\
\text { Instructional strategy: } \\
\text { case-based } \\
\text { MID: videoconferencing } \\
\text { Interactivity: online discus- } \\
\text { sion (synchronous) }\end{array}$ \\
\hline $\begin{array}{l}\text { Helms, Denson et al. } 2009 \\
\text { [55] }\end{array}$ & $\begin{array}{l}\text { Higher education } \\
\text { Medical students }\end{array}$ & $\begin{array}{l}\text { E-module: Neurology and } \\
\text { Dementia: Psychological } \\
\text { Aspects of Care (with clerk- } \\
\text { ship materials) }\end{array}$ & Total duration NS & $\begin{array}{l}\text { Delivery method: fully } \\
\text { online } \\
\text { Instructional strategy: NS } \\
\text { MID: video; text; external } \\
\text { learning resources } \\
\text { Interactivity: NS }\end{array}$ \\
\hline $\begin{array}{l}\text { Hobday, Savik, and Gaugler } \\
2010 \text { [57] }\end{array}$ & $\begin{array}{l}\text { Long-term care } \\
\text { Direct care workers }\end{array}$ & $\begin{array}{l}\text { Internet-based multimedia } \\
\text { education program: demen- } \\
\text { tia training resource }\end{array}$ & $\begin{array}{l}3 \text { prototype modules } \\
\text { Module duration: } 1 \mathrm{~h}\end{array}$ & $\begin{array}{l}\text { Delivery method: Fully } \\
\text { Online } \\
\text { Instructional strategy: NS } \\
\text { MID: video; text; audio } \\
\text { Interactivity: NS }\end{array}$ \\
\hline Hobday et al. 2010 [58] & $\begin{array}{l}\text { Long-term care } \\
\text { Nurse assistants }\end{array}$ & $\begin{array}{l}\text { Internet-based, interac- } \\
\text { tive, multimedia dementia } \\
\text { educational program }\end{array}$ & $\begin{array}{l}4 \text { modules } \\
\text { Module duration: } 1 \mathrm{~h}\end{array}$ & $\begin{array}{l}\text { Delivery method: fully } \\
\text { online } \\
\text { Instructional strategy: NS } \\
\text { MID: video; text; audio-narra- } \\
\text { tion; graphics } \\
\text { Interactivity: NS }\end{array}$ \\
\hline $\begin{array}{l}\text { Hobday, Gaugler and Mittel- } \\
\text { man } 2017 \text { [56] }\end{array}$ & $\begin{array}{l}\text { Hospital } \\
\text { Nursing assistants and allied } \\
\text { hospital workers }\end{array}$ & $\begin{array}{l}\text { CARES }^{\circledR} \text { dementia-friendly } \\
\text { hospital program: online } \\
\text { dementia training program }\end{array}$ & $\begin{array}{l}4 \text { modules } \\
\text { Total duration NS }\end{array}$ & $\begin{array}{l}\text { Delivery method: fully } \\
\text { online } \\
\text { Instructional strategy: } \\
\text { case-based } \\
\text { MID: video; audio-narrated } \\
\text { text; text considers literacy } \\
\text { levels; graphics } \\
\text { Interactivity: NS }\end{array}$ \\
\hline $\begin{array}{l}\text { Irvine, A. B., Bourgeois et al. } \\
2007 \text { [17] }\end{array}$ & $\begin{array}{l}\text { Long-term care } \\
\text { Nurse aides }\end{array}$ & $\begin{array}{l}\text { Interactive multimedia } \\
\text { program: professional } \\
\text { dementia care (managing } \\
\text { aggression) }\end{array}$ & Total duration NS & $\begin{array}{l}\text { Delivery method: fully } \\
\text { online } \\
\text { Instructional strategy: } \\
\text { video-modelling } \\
\text { MID: video; text considers } \\
\text { literacy levels; narration; } \\
\text { graphics } \\
\text { Interactivity: NS }\end{array}$ \\
\hline
\end{tabular}


Table 5 (continued)

\begin{tabular}{|c|c|c|c|c|}
\hline Study & Setting/participants & Programme & Duration & Programme characteristics \\
\hline $\begin{array}{l}\text { Irvine, A. Blair, Beaty et al. } \\
2013 \text { [59] }\end{array}$ & $\begin{array}{l}\text { Long-term care } \\
\text { Non-direct care staff includ- } \\
\text { ing nurses }\end{array}$ & $\begin{array}{l}\text { Internet dementia-training } \\
\text { program }\end{array}$ & $\begin{array}{l}5 \text { modules } \\
2 \mathrm{~h} \text { to complete all modules }\end{array}$ & $\begin{array}{l}\text { Delivery method: fully } \\
\text { online } \\
\text { Instructional strategy: } \\
\text { video-modelling } \\
\text { MID: video; text considers } \\
\text { literacy levels; narration } \\
\text { Interactivity: NS }\end{array}$ \\
\hline Jones, Moyle 2016 [60] & $\begin{array}{l}\text { Long-term care } \\
\text { Nurses, care workers, and } \\
\text { students }\end{array}$ & $\begin{array}{l}\text { Online self-directed e-learn- } \\
\text { ing education intervention } \\
\text { (based on the sexualities } \\
\text { and dementia education } \\
\text { resource for health profes- } \\
\text { sionals) }\end{array}$ & $\begin{array}{l}4 \text { modules } \\
\text { Module duration: } 1 \mathrm{~h}\end{array}$ & $\begin{array}{l}\text { Delivery method: fully } \\
\text { online } \\
\text { Instructional strategy: } \\
\text { case-based } \\
\text { MID: NS } \\
\text { Interactivity: NS }\end{array}$ \\
\hline $\begin{array}{l}\text { Kimzey, Mastel-Smith et al. } \\
2016 \text { [61] }\end{array}$ & $\begin{array}{l}\text { Higher education } \\
\text { Nursing students }\end{array}$ & $\begin{array}{l}\text { Alzheimer's disease online } \\
\text { module }\end{array}$ & Total duration NS & $\begin{array}{l}\text { Delivery method: fully } \\
\text { online } \\
\text { Instructional strategy: NS } \\
\text { MID: NS } \\
\text { Interactivity: NS }\end{array}$ \\
\hline Luconi 2008 [62] & $\begin{array}{l}\text { Primary care } \\
\text { Family physicians }\end{array}$ & $\begin{array}{l}\text { Early Alzheimer's disease } \\
\text { program: web-based con- } \\
\text { tinuing medical education } \\
\text { program }\end{array}$ & $\begin{array}{l}8 \text { modules } \\
\text { completed over } 6 \text { months }\end{array}$ & $\begin{array}{l}\text { Delivery method: fully } \\
\text { online } \\
\text { Instructional strategy: } \\
\text { case-based; practice-based } \\
\text { learning method } \\
\text { MID: text; external learning } \\
\text { resources } \\
\text { Interactivity: online } \\
\text { discussions (asynchronous); } \\
\text { moderated }\end{array}$ \\
\hline $\begin{array}{l}\text { Tomaz, Jose Batista Cisne, } \\
\text { Mamede et al. } 2015 \text { [66] }\end{array}$ & $\begin{array}{l}\text { Primary care } \\
\text { Family physicians }\end{array}$ & $\begin{array}{l}\text { Online PBL: clinical } \\
\text { approach for elderly with } \\
\text { dementia }\end{array}$ & $\begin{array}{l}120 \text { h ( } 100 \text { h distance } \\
\text { and } 20 \text { face-to-face) over } \\
12 \text { weeks }\end{array}$ & $\begin{array}{l}\text { Delivery method: blended } \\
\text { learning } \\
\text { Instructional strategy: case- } \\
\text { based; PBL } \\
\text { MID: video; face-to-face } \\
\text { Interactivity: face-to-face } \\
\text { discussion; online discussions } \\
\text { (synchronous; asynchronous) }\end{array}$ \\
\hline $\begin{array}{l}\text { Vollmar, Mayer et al. } 2010 \\
\text { [68] }\end{array}$ & $\begin{array}{l}\text { Primary care } \\
\text { General practitioners }\end{array}$ & $\begin{array}{l}\text { Online modules: presenta- } \\
\text { tion of a dementia guideline }\end{array}$ & $\begin{array}{l}\text { Estimated average activity } \\
\text { duration: } \\
83 \text { (15 to 200) min }\end{array}$ & $\begin{array}{l}\text { Delivery method: blended } \\
\text { learning } \\
\text { Instructional strategy: } \\
\text { case-based } \\
\text { MID: online NOS } \\
\text { Interactivity: face-to-face } \\
\text { discussions }\end{array}$ \\
\hline $\begin{array}{l}\text { Westmoreland, Counsell } \\
\text { et al. } 2010 \text { [69] }\end{array}$ & $\begin{array}{l}\text { Higher education } \\
\text { Medical residents }\end{array}$ & $\begin{array}{l}\text { Dementia education } \\
\text { module within a web-based } \\
\text { geriatrics training program }\end{array}$ & Total duration NS & $\begin{array}{l}\text { Delivery method: fully } \\
\text { online } \\
\text { Instructional strategy: } \\
\text { case-based } \\
\text { MID: video; text; graphics } \\
\text { Interactivity: NS }\end{array}$ \\
\hline
\end{tabular}

MID Mode of information delivery, NOS Not otherwise specified, NS Not stated/specified, PBL Problem-based learning

${ }^{a}$ The final module was delivered through a face-to-face presentation-not otherwise classified as blended learning

${ }^{b}$ Multiple classifications used. Classified as fully online dementia education as authors report the efficacy of an 'online module' and conclude the utility of online e-modules

'This study included substantial face-to-face learning $(20 \mathrm{~h})$ and was classified as blended learning on this basis

The key programmes characteristics in ODE are summarised in Table 5. Programme characteristics are unlikely to be fully representative due to underreporting in the primary studies.

\section{Effect of ODE on learner satisfaction}

None of the included studies reported on learners' reaction to ODE programmes using experimental or quasiexperimental methods. Ten studies included reports of 
learner satisfaction in additional narrative or survey evaluations $[14,17,53,54,56-60,62]$.

\section{Effect of ODE on knowledge}

Fifteen studies evaluated the effects of ODE programmes on learners' knowledge [14, 17, 52-54, 56-62, 66, 68, 69]. Four studies described validated instruments for outcome measurement: the Alzheimer's Disease Knowledge Scale [61], the Ageing Sexual Knowledge and Attitudes Scale [60], the Communication Knowledge Scale [52], and the Dementia Knowledge Assessment Scale [14]. Only one instrument, a Chinese version of the Communication Knowledge Scale, was validated for the study context [52]. Eight studies created study specific instruments for outcome measurement and provided evidence of validation $[53,54,56-58,62,66,69]$. The validity of outcome measures were not reported in three studies $[17,59,68]$. Where outcome measures were reported, there was inconsistent reporting of validity and reliability. One study used more than one outcome measure [62]. Twelve studies were from practice settings $[14,17,52,54$, $56-60,62,66,68]$ and three were from higher education $[53,61,69]$. Practice-based studies involved practitioners from long-term care $[17,52,57-60]$, primary care [14, $62,66,68]$, and hospital [56]. One study involved practitioners from a variety of healthcare settings [54]. Three practice-based studies compared outcomes between intervention and control/comparator groups $[17,66,68]$. Modest knowledge gains were reported among GPs who completed a blended learning programme consisting of online modules and structured discussions compared to those who attended a traditional lecture and structured discussions; however, the difference between the groups was not statistically significant [68]. Knowledge gains were significantly greater among long-term care practitioners who participated in an internet-based training using video-modelling and mastery learning compared to a control group who did not participate in training [17]. Family physicians demonstrated significantly improved knowledge following participation in a blended approach to problem-based learning compared to a control group who did not receive the training [66]. Nine of the practice-based studies did not involve comparator/control groups and evaluated differences in practitioners' knowledge before and after ODE [14, 52, 54, 56-60, 62]. All of these studies demonstrated improvements in practitioner knowledge following ODE. Three studies of ODE were conducted in higher education settings involving either nursing students $[53,61]$ or medical residents [69]. All studies from higher education compared outcomes between intervention and control/comparator groups. Statistically significant knowledge gains were established among medical residents where a web-based dementia education module was compared to paper-based learning [69]. Knowledge gains were significantly greater among nursing students who completed an ODE programme compared to a control group who did not [53]. In the third study, nursing students demonstrated modest post-test knowledge gains following an Alzheimer's Disease online module whereas the control group, who did not receive any form of dementia training, did not. It is of note that this study involved an additional experiential arm of students who completed learning in practice. The experiential group demonstrated greater knowledge gains compared to the ODE group. Only the findings for the experiential group were statistically significant [61]. The key study characteristics and findings for the knowledge-based outcomes following ODE are summarised in Table 6.

\section{Effect of ODE on skills}

Five studies evaluated the impact of ODE on learners' skills $[17,52,55,59,66]$. Outcomes were assessed using a variety of measures. One study reported reliable measures to assess the application of the mini-mental state exam (MMSE) and skills in differential diagnosis [66]. In another study, the research team developed and validated the Communication Competency Scale [52]. The remaining studies did not provide evidence of validated outcome measures $[17,55]$ although one study reported alpha statistics providing some evidence of reliability [59]. Four studies used more than one outcome measure $[17,55$, $59,66]$. Four studies were conducted in the practice setting $[17,52,59,66]$. The practice-based studies involved practitioners from long-term care [17, 52, 59] and primary care [66]. Two practice-based studies compared outcomes between intervention and control/comparator groups $[17,66]$. Family physicians who participated in the blended approach to problem-based learning demonstrated significantly improved skills in differential diagnosis and mini-mental state examinations when compared to a control group who did not receive the training [66]. Nurse aides who completed the internet-based training using video-modelling demonstrated improved self-efficacy regarding distressed resident behaviours when compared to a control group who did not receive the training [17]. We classified self-efficacy as a 'skill' as it is often concerned with judgments of how well one can execute a course of action required to deal with situations [70]; furthermore, it has received similar classification in the existing literature on TEDE [65]. The other two practicebased studies did not involve comparator/control groups and evaluated differences in practitioners' skills before and after ODE [52, 59]; however, only one study demonstrated improvements in practitioners' skills [52]. One study was from higher education and involved medical 
Table 6 Study characteristics and findings for knowledge-based outcomes following online dementia education

\begin{tabular}{|c|c|c|c|c|c|}
\hline Study & Setting & Study design & Comparator & Measure & Results \\
\hline $\begin{array}{l}\text { Bentley, Kerr et al. } 2019 \\
\text { [14] }\end{array}$ & Practice & Before-after & NA & DKAS & Evidence of effect ${ }^{a}$ \\
\hline $\begin{array}{l}\text { Chao, Kaas et al. } 2016 \\
\text { [52] }\end{array}$ & Practice & Repeated measures & NA & CKS-C & Evidence of effect ${ }^{b}$ \\
\hline $\begin{array}{l}\text { Cobbett, Redmond } \\
\text { et al. } 2016 \text { [53] }\end{array}$ & $\mathrm{HE}$ & Controlled before-after & $\begin{array}{l}\text { Did not participate in } \\
\text { online training }\end{array}$ & $\mathrm{MCQ}^{\mathrm{c}}$ & Evidence of effect \\
\hline $\begin{array}{l}\text { De Witt Jansen, Brazil } \\
\text { et al. } 2018 \text { [54] }\end{array}$ & Practice & Before-after & NA & ECHO Questionnaire $^{d}$ & Evidence of effect \\
\hline $\begin{array}{l}\text { Hobday, Savik, and } \\
\text { Gaugler } 2010 \text { [57] }\end{array}$ & Practice & Before-after & NA & Knowledge Inventory & Evidence of effect \\
\hline Hobday et al. 2010 [58] & Practice & Before-after & NA & $\begin{array}{l}\text { Dementia Care Knowl- } \\
\text { edge }\end{array}$ & Evidence of effect \\
\hline $\begin{array}{l}\text { Hobday, Gaugler and } \\
\text { Mittelman } 2017 \text { [56] }\end{array}$ & Practice & Before-after & NA & $\begin{array}{l}\text { Dementia Care Knowl- } \\
\text { edge }\end{array}$ & Evidence of effect \\
\hline $\begin{array}{l}\text { Irvine, A. B., Bourgeois } \\
\text { et al. } 2007[17]\end{array}$ & Practice & Randomised trial & $\begin{array}{l}\text { Did not participate in } \\
\text { training }\end{array}$ & VST: knowledge & Evidence of effect \\
\hline $\begin{array}{l}\text { Irvine, A. Blair, Beaty } \\
\text { et al. } 2013 \text { [59] }\end{array}$ & Practice & Before-after & NA & VST: knowledge & Evidence of effect ${ }^{\mathrm{e}}$ \\
\hline Jones, Moyle 2016 [60] & Practice & Before-after & NA & ASKAS: knowledge & Evidence of effect \\
\hline $\begin{array}{l}\text { Kimzey, Mastel-Smith } \\
\text { et al. } 2016 \text { [61] }\end{array}$ & HE & Controlled before-after & $\begin{array}{l}\text { Usual practice; Experi- } \\
\text { ential learning }\end{array}$ & ADKS & No evidence of effect \\
\hline Luconi 2008 [62] & Practice & Repeated measures & NA & MCQ; clinical cases & $\begin{array}{l}\text { Partial evidence of } \\
\text { effect }^{\mathrm{b}} \text { (multiple outcome } \\
\text { measures) }\end{array}$ \\
\hline $\begin{array}{l}\text { Tomaz, Jose Batista } \\
\text { Cisne, Mamede et al. } \\
2015 \text { [66] }\end{array}$ & Practice & Controlled before-after & $\begin{array}{l}\text { Did not receive train- } \\
\text { ing }\end{array}$ & Knowledge test & Evidence of effect \\
\hline $\begin{array}{l}\text { Vollmar, Mayer et al. } \\
2010 \text { [68] }\end{array}$ & Practice & Randomised trial & Traditional lecture & Knowledge test & No evidence of effect \\
\hline $\begin{array}{l}\text { Westmoreland, Coun- } \\
\text { sell et al. } 2010 \text { [69] }\end{array}$ & $\mathrm{HE}$ & Randomised trial & Paper-based learning & Knowledge test & Evidence of effect \\
\hline
\end{tabular}

ADKS Alzheimer's Disease Knowledge Scale, ASKAS Ageing Sexual Knowledge and Attitudes Scale, CKS-C Communication Knowledge Scale-Chinese version, DKAS Dementia Knowledge Assessment Scale, HE Higher education, MCQ Multiple choice question, NA Not applicable, VST Video situation test

${ }^{\text {a }}$ Based on descriptive statistics

${ }^{\mathrm{b}}$ Based on first time point following training

${ }^{c}$ Measured comprehension, application, and critical thinking

${ }^{\mathrm{d}}$ Measured knowledge and self-efficacy

e The findings related to nurses only

Table 7 Study characteristics and findings for skills-based outcomes following online dementia education

\begin{tabular}{|c|c|c|c|c|c|}
\hline Study & Setting & Study design & Comparator & Measure & Results \\
\hline Chao, Kaas et al. 2016 [52] & Practice & Repeated measures & NA & CCS & Evidence of effect ${ }^{a}$ \\
\hline $\begin{array}{l}\text { Helms, Denson et al. } 2009 \\
\text { [55] }\end{array}$ & $\mathrm{HE}$ & Non-randomised trial & $\begin{array}{l}\text { Did not participate in } \\
\text { e-module }\end{array}$ & $\begin{array}{l}\text { OSCE clinical note score; } \\
\text { OSCE performance }\end{array}$ & Evidence of effect \\
\hline $\begin{array}{l}\text { Irvine, A. B., Bourgeois et al. } \\
2007 \text { [17] }\end{array}$ & Practice & Randomised trial & $\begin{array}{l}\text { Did not participate in } \\
\text { training }\end{array}$ & $\begin{array}{l}\text { Self-efficacy; VST: Self } \\
\text { efficacy }\end{array}$ & Evidence of effect \\
\hline $\begin{array}{l}\text { Irvine, A. Blair, Beaty et al. } \\
2013 \text { [59] }\end{array}$ & Practice & Before-after & NA & $\begin{array}{l}\text { Self-efficacy; VST: Self } \\
\text { efficacy }\end{array}$ & No evidence of effect ${ }^{b}$ \\
\hline $\begin{array}{l}\text { Tomaz, Jose Batista Cisne, } \\
\text { Mamede et al. } 2015 \text { [66] }\end{array}$ & Practice & Controlled before-after & Did not receive training & DD; MMSE & Evidence of effect \\
\hline
\end{tabular}

CCS Communication Competency Scale, DD Differential diagnosis, HE Higher education, MMSE Mini-mental state examination, NA Not applicable, OSCE Objective structured clinical examination, VST Video situation tests

a Based on first time point following training

${ }^{\mathrm{b}}$ The findings relate to nurses only 
Table 8 Study characteristics and findings for studies reporting attitudinal change following online dementia education

\begin{tabular}{|c|c|c|c|c|c|}
\hline Study & Setting & Study design & Comparator & Measure & Results \\
\hline Bentley, Kerr et al. 2019 [14] & Practice & Before-after & NA & GPACS-D & Evidence of effect ${ }^{a}$ \\
\hline Chao, Kaas et al. 2016 [52] & Practice & Repeated measures & NA & CSAS-C & No evidence of effect ${ }^{b}$ \\
\hline $\begin{array}{l}\text { Irvine, A. B., Bourgeois et al. } \\
2007 \text { [17] }\end{array}$ & Practice & Randomised trial & $\begin{array}{l}\text { Did not participate in train- } \\
\text { ing }\end{array}$ & $\begin{array}{l}\text { Attitudes; behavioural Inten- } \\
\text { tions }\end{array}$ & Evidence of effect \\
\hline $\begin{array}{l}\text { Irvine, A. Blair, Beaty et al. } \\
2013 \text { [59] }\end{array}$ & Practice & Before-after & NA & $\begin{array}{l}\text { Attitudes; behavioural inten- } \\
\text { tions }\end{array}$ & $\begin{array}{l}\text { Partial evidence of } \\
\text { effect }^{c} \text { (multiple out- } \\
\text { come measures) }\end{array}$ \\
\hline Jones, Moyle 2016 [60] & Practice & Before-after & NA & ASKAS: attitude; SAID & Evidence of effect \\
\hline $\begin{array}{l}\text { Kimzey, Mastel-Smith et al. } \\
2016 \text { [61] }\end{array}$ & $\mathrm{HE}$ & Controlled before-after & $\begin{array}{l}\text { Usual practice; experiential } \\
\text { learning }\end{array}$ & DAS & No evidence of effect \\
\hline
\end{tabular}

ASKAS Ageing Sexual Knowledge and Attitudes Scale, CSAS-C Communications Skills Attitudes Scale-Chinese version, DAS Dementia Attitudes Scale, GPACS-D Confidence and Attitudes Towards Dementia Scale, HE Higher education, NA not applicable, SAID Staff Attitudes about Intimacy and Dementia Survey

${ }^{\text {a }}$ Based on descriptive statistics

${ }^{b}$ Based on first time point following training

${ }^{c}$ The findings related to nurses only

students who completed an e-module on psychosocial aspects of dementia care and subsequently performed better in an OSCE when compared to a control group who did not participate in the training [55]. The key study characteristics and findings for the skills-based outcomes following ODE are summarised in Table 7.

\section{Effect of ODE on attitudes}

Six studies evaluated the impact of ODE on learners' attitudes [14, 17, 52, 59-61]. Four studies used previously validated instruments for outcome measures: the Confidence and Attitudes Towards Dementia Scale [14], the Communications Skills Attitudes Scale [52], the Aging Sexual Knowledge and Attitude Scale [60], and the Dementia Attitudes Scale [61]. The Communications Skills Attitudes Scale-Chinese version was validated for the study context [52]. One study used the Staff Attitudes about Intimacy and Dementia Survey although the psychometric properties were undetermined [60]. The remaining studies used programmespecific outcome measures; one was unvalidated [17] and one provided evidence of reliability only [59]. Three studies used more than one outcome measure [17, 59, $60]$. Five studies were conducted in the practice setting $[14,17,52,59,60]$. The practice-based studies involved practitioners from long-term care $[17,52,59,60]$ and primary care [14]. Only one of the practice-based studies compared outcomes between intervention and control/comparator groups.

The nurse aides who completed internet-based training using video-modelling demonstrated improved attitudes and behavioural intentions regarding distressed resident behaviours when compared to a control group who did not receive the training [17]. Behavioural interventions were classified as 'attitudes' as, according to the Theory of Reasoned Action, attitudes are postulated as direct determinants of behavioural intentions [71]. Four practice-based studies did not involve comparator/control groups and evaluated differences in practitioners' attitudes before and after ODE [14, 52, $59,60]$. Improved staff attitudes were observed following the training in all but one study [52].

One study was conducted in higher education and compared attitudinal change among nursing students who either completed an ODE programme or received no dementia specific intervention. Modest and nonsignificant attitudinal change was observed in both groups. It is of note that this study involved an additional arm comprising of students who completed experiential learning in practice. The experiential group demonstrated statistically significant improvements in attitudes toward people with dementia [61]. The key study characteristics and findings for the studies reporting attitudinal change following ODE are summarised in Table 8.

\section{Effect of ODE on behaviours}

Two studies evaluated the impact of ODE on learners' behaviours [52, 69]. One study used multiple outcome measures and did not report on the validation of the measures used [69]. The other study reported a reliable and valid outcome measure [52]. The studies were carried out in long-term care [52] and higher education [69]. In long-term care, the frequency in which nurses assessed their patients' communication ability was assessed before and after the blended learning programme on communication between nurses and patients with dementia. The results indicated that a 
Table 9 Study characteristics and findings for behaviour-based outcomes following online dementia education

\begin{tabular}{|c|c|c|c|c|c|}
\hline Study & Setting & Study Design & Comparator & Measure & Results \\
\hline Chao, Kaas et al. 2016 [52] & Practice & Repeated measures & NA & PREAS & Evidence of effect ${ }^{a}$ \\
\hline $\begin{array}{l}\text { Westmoreland, Counsell } \\
\text { et al. } 2010 \text { [69] }\end{array}$ & $\mathrm{HE}$ & Randomised trial & Paper-based learning & $\begin{array}{l}\text { Chart abstraction; encoun- } \\
\text { ter checklist; EOES }\end{array}$ & $\begin{array}{l}\text { Partial evidence of } \\
\text { effect (multiple outcome } \\
\text { measures) }\end{array}$ \\
\hline
\end{tabular}

EOES Electronic Order Entry Score, HE Higher education, NA Not applicable, PREAS Patients Receptive and Expressive Ability Assessment Scale

${ }^{a}$ Based on first time point following training

higher frequency of assessments was conducted following the training [52]. In higher education, postgraduate medical residents' behaviours were evaluated in interactions with unannounced standardised patients in the practice setting following the web-based dementia education module. Outcome measures included an encounter checklist, chart abstraction scores, and treatment orders placed on an electronic medical record system. Only residents' chart abstraction scores were significantly better when compared with a comparator group that participated in paper-based learning [69]. The key study characteristics and findings for the behaviour-based outcomes following ODE are summarised in Table 9.

\section{Effect of $O D E$ on results}

The study that evaluated blended learning to improve communication between nurses and patients with dementia also included patient-level outcomes. This study evaluated differences in resident behaviours before and after practitioners received the training using two outcome measures: the Revised Memory and Behaviour Problems Checklist-Chinese version and the Cornell Scale for Depression in DementiaChinese version (CSDD-C). Validation of the CSDD-C was reported and both measures were reliability tested in context. The findings suggested that depressive symptoms but not behavioural problems improved at 4 weeks after the training; however, the findings were not statistically significant for either of the outcomes measured [52].

\section{Computer-based dementia education programmes}

Two non-networked CBDE programmes were from practice-based settings $[1,67]$ and three were from higher education [63-65]. The CBDE programmes were either dementia training delivered on a CD-ROM $[1,65]$ or were computer-based simulation activities $[63,64,67]$. CD-ROM trainings were described as educational tutorials [1] or multimedia training [65]. Simulation activities included a clinic simulator involving virtual patients [63], a video simulator modelling appropriate levels of dressing assistance [67], and computer-based branching path simulation (BPS). BPS is interactive learning tool that can develop critical thinking skills and decision-making capability among learners [64]. Key characteristics of the CBDE programmes are summarised in Table 10.

\section{Effect of CBDE on learner satisfaction}

Learner satisfaction was included in an evaluation of the clinic simulator involving virtual patients with dementia. Medical students' motivation for learning was measured using a Japanese language version of the Attention, Relevance, Confidence, and Satisfaction (ARCS) motivational model. Mean values increased significantly in all four ARCS categories after learners' experience with the simulator [63]. In a study of CBDE using a multimedia CDROM, nursing students reported statistically significant improvements in pre- to post-training ratings for utility and comfort with computer-based training in general [65]. Four studies of CBDE reported on aspects of learner satisfaction in additional descriptive narrative or survey evaluations [63-65, 67].

\section{Effect of CBDE on knowledge}

Two studies evaluated the effects of CBDE programmes on learners' knowledge [63, 65]. Both studies measured outcomes using a knowledge test. One of the measures was a study specific evaluation tool with evidence of validation [63]. The validity of the other measure was not reported [65]. Both studies were from higher education. One study compared the before and after scores of medical students who participated in a clinic simulator with the scores of a control group who did not participate in the simulator experience. The intervention group had significantly higher scores after the training [63]. The other study did not involve a control group and compared nursing students' knowledge before and after a multimedia training CD-ROM [65]. A statistically significant increase in learners' knowledge was observed following the training.

\section{Effect of CBDE on skills}

Two studies evaluated the impact of CBDE programmes on learners' skills $[64,65]$. One study 
Table 10 Key characteristics of computer-based dementia education programmes

\begin{tabular}{|c|c|c|c|c|}
\hline Study & Setting/participants & Programme & Duration & Programme characteristics \\
\hline Downs, Turner et al. 2006 [1] & $\begin{array}{l}\text { Primary care } \\
\text { GP practices }\end{array}$ & $\begin{array}{l}\text { Educational tutorial on } \\
\text { CD-ROM }\end{array}$ & Total duration: NS & $\begin{array}{l}\text { Delivery methods: CD-ROM } \\
\text { Instructional strategy: case- } \\
\text { based } \\
\text { MID: electronic book (hyper- } \\
\text { text indexing system) }\end{array}$ \\
\hline $\begin{array}{l}\text { Matsumura, Shinno et al. } \\
2018 \text { [63] }\end{array}$ & $\begin{array}{l}\text { Higher education } \\
\text { Medical students }\end{array}$ & $\begin{array}{l}\text { Clinic simulator with virtual } \\
\text { patients (alongside conven- } \\
\text { tional learning) }\end{array}$ & Simulator duration: $0.75 \mathrm{~h}$ & $\begin{array}{l}\text { Delivery methods: Windows } \\
7 \text { Operating System } \\
\text { Instructional strategy: Simu- } \\
\text { lated learning; case-based } \\
\text { MID: 3D simulation; text; } \\
\text { video }\end{array}$ \\
\hline Rababa, Masha'al 2020 [64] & $\begin{array}{l}\text { Higher education } \\
\text { Nursing students }\end{array}$ & $\begin{array}{l}\text { Computer-based BPS for } \\
\text { pain management in peo- } \\
\text { ple with dementia }\end{array}$ & $\begin{array}{l}6 \text { sessions } \\
\text { Session duration: 1-h dura- } \\
\text { tion }\end{array}$ & $\begin{array}{l}\text { Delivery methods: } \\
\text { computer-based } \\
\text { Instructional strategy: BPS; } \\
\text { case-based } \\
\text { MID: NS }\end{array}$ \\
\hline Ruiz, Smith et al. 2006 [65] & $\begin{array}{l}\text { Higher education } \\
\text { Nursing students }\end{array}$ & $\begin{array}{l}\text { Multimedia training CD- } \\
\text { ROM: Alzheimer's and other } \\
\text { Dementias }\end{array}$ & $\begin{array}{l}7 \text { modules } \\
\text { Module duration: } \\
20-30 \text { min }\end{array}$ & $\begin{array}{l}\text { Delivery methods: } \mathrm{CD}-\mathrm{ROM} \\
\text { Instructional strategy: NS } \\
\text { MID: video; text; graphics; } \\
\text { audio }\end{array}$ \\
\hline Tsai, Kitch et al. 2018 [67] & $\begin{array}{l}\text { Long-term care } \\
\text { Nursing Assistants and } \\
\text { Residents }\end{array}$ & $\begin{array}{l}\text { Computer-based simula- } \\
\text { tion-appropriate level } \\
\text { of dressing assistance for } \\
\text { people with dementia (with } \\
\text { conventional learning) }\end{array}$ & Simulator duration: $2 \mathrm{~h}$ & $\begin{array}{l}\text { Delivery methods: tablet } \\
\text { device } \\
\text { Instructional strategy: } \\
\text { simulated learning; video } \\
\text { modelling } \\
\text { MID: video; text }\end{array}$ \\
\hline
\end{tabular}

BPS Branching path simulation, MID Mode of information delivery, NS Not stated/specified

measured outcomes using the previously validated Critical Thinking Self-Assessment Scale (CTSAS) [64]. The other used a 7-item questionnaire to measure self-reported self-efficacy in dementia care skills and did not provide evidence of validation [65]. Both studies were from higher education and involved nursing students. One study compared critical thinking skills between an intervention group with a control group before and after computer-based BPS for pain management in people with dementia. After the training, CTSAS scores in the intervention group were significantly higher than the control group [64]. The other study did not involve a control group and compared nursing students' self-efficacy in dementia care before and after a multimedia training CD-ROM. Selfreported self-efficacy scores increased significantly after the training [65].

\section{Effect of CBDE on attitudes}

The study reporting on the multimedia training CDROM also measured attitudinal change among nursing students before and after the training. The measurement tool was a single questionnaire item designed to assess participants desire to provide care to people with dementia. Participant responses indicated an increased desire to provide care to people with dementia after the training. The findings were statistically significant [65].

\section{Effect of CBDE on behaviours}

Two studies assessed practitioner behaviours following CBDE $[1,67]$. One study reported on the reliability of the outcome measure [67]. In the other study, practitioner behaviour was assessed using data from an electronic clinical records system [1]. Both studies were from practice settings; one from long-term care [67] and the other from primary care [1]. In long-term care, nursing assistants learned appropriate levels of dressing assistance to give their residents using a video simulator. Following the simulation activity, the nursing assistants provided more appropriate levels of assistance compared to a control group; however, the comparative difference was not statistically significant [67]. In primary care, dementia diagnosis rates among primary care practitioners were assessed following engagement with an electronic tutorial on CD-ROM, practice-based workshops, or decision support software. When compared to a control group, only the workshop and decision support interventions resulted in significant improvements in diagnosis rates. The study also reported on concordance rates with clinical guidelines for diagnosis and management of dementia. There were no significant differences between the groups studied [1].

\section{Effect of CBDE on results}

Tsai et al. [67] also evaluated wider results (patientlevel outcomes) following the video simulator activity. 
Table 11 Study characteristics and findings for all outcomes following computer-based dementia education

\begin{tabular}{|c|c|c|c|c|c|c|}
\hline Study & Setting & Study design & Comparator & Outcome & Measure & Results \\
\hline $\begin{array}{l}\text { Downs, Turner et al. } \\
2006 \text { [1] }\end{array}$ & Practice & Randomised trial & $\begin{array}{l}\text { Workshop; decision } \\
\text { support; } \\
\text { control (no training } \\
\text { intervention) }\end{array}$ & Behaviours & $\begin{array}{l}\text { Detection rates; } \\
\text { concordance with } \\
\text { guidelines }\end{array}$ & No evidence of effect \\
\hline $\begin{array}{l}\text { Matsumura, Shinno } \\
\text { et al. } 2018 \text { [63] }\end{array}$ & $\mathrm{HE}$ & $\begin{array}{l}\text { Controlled before- } \\
\text { after }\end{array}$ & $\begin{array}{l}\text { Did not participate } \\
\text { in clinic simulator } \\
\text { activity }\end{array}$ & $\begin{array}{l}\text { Satisfaction } \\
\text { Knowledge }\end{array}$ & $\begin{array}{l}\text { ARCS } \\
\text { Knowledge test }\end{array}$ & $\begin{array}{l}\text { Evidence of effect } \\
\text { Evidence of effect }\end{array}$ \\
\hline $\begin{array}{l}\text { Rababa, Masha'al } \\
2020 \text { [64] }\end{array}$ & $\mathrm{HE}$ & $\begin{array}{l}\text { Controlled before- } \\
\text { after }\end{array}$ & Traditional lectures & Skills & CTSAS & Evidence of effect \\
\hline $\begin{array}{l}\text { Ruiz, Smith et al. } \\
2006 \text { [65] }\end{array}$ & $\mathrm{HE}$ & Before-after & NA & $\begin{array}{l}\text { Satisfaction } \\
\text { Knowledge } \\
\text { Skills } \\
\text { Attitudes }\end{array}$ & $\begin{array}{l}\text { PPT } \\
\text { Knowledge test } \\
\text { Self-efficacy test } \\
\text { Attitude test }\end{array}$ & $\begin{array}{l}\text { Evidence of effect } \\
\text { Evidence of effect } \\
\text { Evidence of effect } \\
\text { Evidence of effect }\end{array}$ \\
\hline $\begin{array}{l}\text { Tsai, Kitch et al. } \\
2018 \text { [67] }\end{array}$ & Practice & $\begin{array}{l}\text { Controlled before- } \\
\text { after }\end{array}$ & $\begin{array}{l}\text { Did not participate } \\
\text { in video simulator } \\
\text { activity }\end{array}$ & $\begin{array}{l}\text { Behaviours } \\
\text { Results }\end{array}$ & $\begin{array}{l}\text { Level of dressing } \\
\text { assistance } \\
\text { BDPS }\end{array}$ & $\begin{array}{l}\text { No evidence of effect } \\
\text { No evidence of effect }\end{array}$ \\
\hline
\end{tabular}

ARCS Attention, Relevance, Confidence, and Satisfaction Motivational Model, BDPS Beck Dressing Performance Scale, CTSAS Critical Thinking Self-Assessment Scale, HE Higher education, NA Not applicable, PPT Pre- and post-training

Residents' dressing performance abilities were measured using the previously validated and reliability tested Beck Dressing Performance Scale. The findings demonstrated pre- to post-training improvements in dressing performance that were greater in the intervention group compared to the control group. The difference between the groups was not statistically significant.

The key study characteristics and findings from nonnetworked CBDE programmes are shown in Table 11.

\section{Sustainability of the learning outcomes}

Four studies used repeated measures or included additional follow-up (FU) data which allowed for inference into the sustainability of learning outcomes following TEDE [52, 55, 62, 68]. Luconi [62] aimed to understand if family physicians' knowledge was maintained following a web-based programme on early Alzheimer's Disease. Pre-test and post-test mean scores from MCQ and clinical cases were compared with scores at one month post-test. Pre-test to FU MCQ scores improved significantly (pre-FU $-4.58, p=0.002$ ); however, the difference from post-test to FU was not statistically significant (post-FU $-0.43, p=0.497$ ). There were no significant differences between the pre-test or post-test to FU scores for problem-solving of clinical cases. Chao et al. [52] reported data for multiple outcomes at 16 weeks following their programme to promote communication between nurses and patients with dementia. This FU data suggested that nurses' communication knowledge and frequency of assessing patients' communication abilities were sustained until at least week 16. Improvements in nurses' attitudes and communication competencies were not apparent over time. The frequency of patients' behavioural and depressive symptoms was observed to decreased at 16 weeks compared to baseline. Vollmar et al. [68] included FU analysis of GPs' knowledge following a blended learning programme at four months following the post-test. The mean pre-test minus FU difference was calculated to be -2.39 in the intervention group and -2.00 in the control group; however, the difference between groups was not significant $(p=0.526)$. Helms [55] adopted a different approach for inference of sustainability of training effects. Data from the intervention group of medical students who participated in a dementia e-module were divided into two subgroups; one subgroup took an OSCE immediately after training (immediate group) and another group who took the OSCE 1 month after training (delayed group). There was a significantly higher OSCE (performance) score in the delayed group compared to the immediate group $(p=0.04)$. The delayed group also had a higher overall clinical note score; however, this difference was not significant $(p=0.24)$.

\section{Discussion}

\section{Main findings}

Evidence suggests that e-learning is as, if not more, effective than traditional education for HSCPs [23, 72]. This review included 21 studies of TEDE for HSCPs and aimed to appraise and synthesise the current evidence from experimental and quasi-experimental research. The review sought to understand if TEDE is beneficial across the outcomes in Kirkpatrick's model. The included studies contained high-levels of statistical heterogeneity which precluded meta-analysis; therefore, narrative synthesis techniques were helpful to demonstrate findings and 
programme characteristics across the individual studies. Dealing with heterogeneity and navigating inconsistent use of terminology are particular challenges in the field of virtual learning [20]. Classification of delivery methods and differentiating between ODE and non-networked CBDE reduced complexity; however, the range of pedagogical characteristics within individual programmes hindered the extent to which training effects could be attributed to overarching delivery methods. Therefore, any inference for knowledge attainment following 'TEDE' was only made possible due to the quantity of individual studies that provided evidence of training effects. Likewise, most studies that included skills-based outcomes reported positive effects due to the training. Evidence for attitudinal change following TEDE was less compelling; however, it is possible that practitioners' attitudes can also be influenced through improved knowledge [73]. Fewer studies reported on the higher-level outcomes (i.e., behaviours and results). More research would be useful to understand if TEDE programmes can support wider organisational outcomes, either directly or when mediated through practitioner learning gains. The review included several studies of TEDE in higher education, long-term care, and primary care. There was limited evidence for TEDE in the acute hospital. This will be a priority area for future research that might also consider how TEDE can support complex organisational demands within busy acute care environments.

\section{Key features for effective TEDE}

Surr et al. [27] identified a number of key features that seem to exist in effective dementia training. Passive teaching and learning methods do not reflect educational best practice and recommendations include active participation in dementia education. TEDE programmes may achieve this by moving away from self-directed approaches that rely on large amounts of textual information and singular instructional modalities toward more multi-modal programmes that include a rich variety of role-relevant instructional strategieswhether in ODE or CBDE. ODE programmes may enable greater levels of activity by virtue of the Web 2.0 technology that allows learners to collaborate and problem solve together [74]. Surr et al. [27] highlighted this need for interactivity in dementia education involving groups of learners and experienced facilitators. Thus, TEDE should aim to meet the individual needs of learners and offer opportunities for collaboration, peer and facilitator support, and group reflective activities [75]. Currently, there is a dearth of evidence on communities of practice and both synchronous and asynchronous communication platforms in ODE. This will be a priority area for future research considering also the recent rapid transition to technology for learning including the accelerated use of chat-based collaboration platforms since COVID-19 [76]. Non-networked CBDE may continue to play a role where learners cannot access online resources, and blended approaches may help to compensate for the pedagogical benefits otherwise derived from interactive learning and Web 2.0. Three studies included blended approaches [52, $66,68]$. One study did not provide evidence for knowledge gains following this approach; however, the training programme was of relatively short duration [68]. Duration of engagement is another important factor in effective dementia education. The total duration of dementia education is relevant as it is likely to influence the training effects [27]. It is less clear if TEDE offers any advantages in terms of time efficiency over traditional methods for dementia education-as both delivery methods are highly context-specific and likely to be influenced by specific pedagogical characteristics and course design [77]. Four studies included evidence for outcome effects over time $[52,55,62,68]$ albeit inference towards the sustainability of training effects was limited due to inconsistent findings between the studies. It is also important that dementia education has relevance to the role and the experience of learners [27]. The current review suggests that this applies, not only to educational content, but to instructional strategies and modes of information delivery in TEDE programmes. For instance, case-based learning was widely applicable across practice and higher educational settings, whereas practice and problem-based learning were described only in the primary care context [62, 66]. Furthermore, studies from long-term care highlighted the need for simplified modes of information delivery [17, 58, 59]. Future research might consider how technology can be harnessed to respond to the specific learning requirements of individuals or groups by exploring adaptive learning technologies for tailored programmes that include material and instructional strategies that are most relevant [78].

\section{Methodological issues in TEDE research}

Despite debate as to whether evidence hierarchies are appropriate frameworks to judge the quality of educational research [79], benefits of randomised trials and controlled studies have been described in similar reviews of TEDE [28]. Reviewers of traditional dementia education endorse randomised trials when considering intervention effectiveness [11]; others highlight challenges associated with randomised trials in healthcare settings [80]; or suggest integrating qualitative methods to deal with complex educational 
interventions [81]. MMAT and MERSQI both included methodological quality judgments based on study design. MMAT highlighted significant confounding bias. Extraneous variables can be problematic in educational research, even using the 'gold standard' randomised trial [82]. MERSQI attributes higher scores to randomised trials, which helps to address issues of selection bias; however, it is noteworthy that control of confounders is not a specific quality indicator. We do not suggest that confounders can be overlooked in TEDE research; however, where study designs do not have robust strategies to circumvent bias arising from confounders, researchers might place a greater emphasis on the acknowledgement of potential confounders and apply controls where it is practical. MMAT appraisal resulted in high levels of uncertainty across many of the other quality domains. This inferred methodological limitations in the primary studies and possible limitations of MMAT to the TEDE research context. For instance, participant representativeness was frequently judged to be unclear; however; this was often as a result of non-probability sampling and intentionally pragmatic methods using accessible learner cohorts. In this context, quality judgements may improve if researchers provide clearer justification for pragmatic methods and acknowledge the associated study limitations including the generalisability of findings. Issues from outcome measurement were covered in both MMAT and MERSQI. For study results to be credible, assessment instruments must be both reliable and valid [83]. In TEDE research, there may be a greater need for validation in context for optimal cohesion between the instructional content and the outcomes measured. In general, MMAT allowed for the appraisal of various study designs common in educational research but may have lacked the specificity required to appraise diverse and often pragmatic educational research methods. MERSQI was a valuable complementary tool as it omitted many issues common in educational research and put additional emphasis on the number of institutions studied, complexity of data analysis techniques, and key educational outcomes-which were well-aligned with Kirkpatrick's model.

\section{Dealing with causality and complexity}

The main advantage of including experimental and quasi-experimental studies in the review is capacity to establish causal inferences between the TEDE programme and the outcomes measured. However, overall conclusions must be treated with caution. The technology alone cannot influence the training effects; rather, it is the pedagogy that the technology enables and supports - and pedagogy differs widely between training programmes [74]. Even where similarities exist (e.g., use of case-based instruction, videos, textual information), the approach is likely to be influenced by intrinsic factors relating to content, quality, and duration, as well as extrinsic factors such as learner engagement. Therefore, these pedagogical strategies need to be considered as layered elements that are nested within programmes and require methods of evaluation that can illicit their specific role and function [84]. Where multiple causal elements exist, methods of evaluation become more complicated, yet it is nonetheless important that these elements are considered in evaluation processes. One way to address this complexity is to employ principles from programme theory which refers to a variety of ways of developing causal models that can link various programme inputs and elements to the intended outcomes [85]. Each element can then be identified and addressed through the pragmatic integration of the most relevant quantitative or qualitative research methods; thereby, gaining a deeper understanding of the more nuanced elements, which, when brought back together, are likely to result in a more meaningful understanding of the 'whole'. Scerri et al. [24] suggested a more inclusive approach to dementia education research towards a richer understanding of complexity and enhanced ecological validity. It is clear that interventional complexity and 'real world' influence cannot be easily eliminated in TEDE research. Future research might therefore shift focus now from internal validity to the most relevant research design typologies that can achieve a more nuanced understanding of these complex educational programmes and context [79].

\section{Limitations}

There are several limitations of this review. Firstly, all attempts were made to conduct a comprehensive literature search and study screening process; however, two references entitled 'resources' could not be located and were excluded at the screening stage. Furthermore, this review only included studies available in the English language. Most titles and abstracts and all full texts were assessed for eligibility by one reviewer which was necessary due to resource limitations. Similarly, one person completed data extraction which may have increased the risk of errors [86]. Quality appraisal was also completed by one reviewer; however, it is worth noting that MERSQI focuses on design issues and is quite objective [50]. It is also worth noting that the overall quality of studies was judged to be 'moderate'-which is consistent with other reviews of TEDE [28]. Second reviewers appraised a proportion of studies using MMAT which mitigated against bias where 
more subjective judgments were involved; however, the application of MMAT to this review context required additional justification as it is traditionally used in systematic mixed studies reviews. It is possible that the exclusion of studies published before 2005 disproportionately limited the evidence for CBDE compared to ODE. This was a pragmatic decision that balanced the need to segregate the two approaches (i.e., to address potential limitations where there may be sub-optimal technological infrastructure for ODE) whilst maintaining a contemporary focus. It is also important to note that CBDE may be reproducible as ODE and vice versa. The review included experimental and quasiexperimental studies and excluded descriptive narrative and survey evaluations of TEDE. This limited the extent to which outcomes based on Kirkpatrick's level 1 (reactions) featured in the review. This limitation was anticipated as participant satisfaction levels are typically evaluated as learner feedback at the end of training programmes which can support future programme development [33, 87]. Satisfaction levels before and after training were reported in two studies of CBDE; however, these related to general concepts such as motivation for learning [63] and comfort with computer-based training [65]. It is not possible to determine pre-test levels of satisfaction with a specific training programme. Post-test comparisons of participant satisfaction between intervention and comparator groups may be achievable; however, the data available did not support this type of analysis. It is important to highlight that many primary studies included complementary reports of participant satisfaction using appropriate descriptive methods of evaluation. Training effects were more appropriately described as improvements in learning gains (knowledge, attitudes, and skills), practitioner behaviours, and wider results due to the training. However, effects were most frequently assumed from controlled studies involving a 'no training' control arm, or from before-after studies-where the before aspect could be considered a 'no training' state. Few studies compared the relative effectiveness of TEDE compared to alternative delivery methods (e.g., traditional education). This will also be a priority focus for future research. Finally, the review did not provide an exhaustive inventory of TEDE programmes as several innovative practices may have been identified in descriptive research that did not satisfy the study selection criteria. Future evidence syntheses that include methods to incorporate these rich sources of information will be crucial to better understand how technology can enable dementia education for the health and social care workforce.

\section{Conclusion}

The current evidence provides several examples where TEDE is beneficial to practitioner development on emotional, as well as intellectual levels, which is crucial for person-centred dementia care. The evidence highlights a need for more emphasis on the teaching and learning methods within TEDE and the requirements of specific learning communities. Establishing innovative active and interactive learning strategies will be integral to the design and development of future training programmes. Future evaluations might explore the relative effectiveness of TEDE compared to traditional dementia education and employ contextually relevant research methods that have capacity to address the challenges presented by these complex educational programmes and multi-component characteristics.

\section{Abbreviations}

BPS: Branching path simulation; CBDE: Computer-based dementia education; FU: Follow-up; HSCP: Health and social care practitioner; MCQ: Multiple choice question; MERSQI: Medical Education Research Study Quality Instrument;

MMAT: Mixed methods appraisal tool; ODE: Online dementia education; OSCE: Objective structured clinical examination; PRISMA: Preferred reporting items for systematic review and meta-analysis; PROSPERO: International prospective register of systematic reviews; SD: Standard deviation; TEDE: Technologyenabled dementia education.

\section{Supplementary Information}

The online version contains supplementary material available at https://doi. org/10.1186/s13643-021-01781-8.

Additional file 1. PRISMA Checklist.

Additional file 2. Search Strategy.

Additional file 3. Sample Data Extraction Form.

Additional file 4. Characteristics of Included Studies.

Additional file 5. Excluded Studies.

Additional file 6. MERSQI Scores (Primary Studies).

Acknowledgements

Not applicable.

Authors' contributions

KM developed the search strategy in collaboration with RP, COM, LM, KS, and CC. KM drafted the manuscript with support from LM, KS, and CCh. KM screened titles and abstracts and performed quality assessment. LM and CC screened $10 \%$ of titles and abstracts. LM and KS appraised $19 \%$ of studies using MMAT. KM conducted data synthesis and analysis with support from LM, KS, and CCh. LM, KS, CCh, and CC were involved in supervising the review process. All authors have read and approved the final manuscript.

\section{Funding}

This systematic review is part of a PhD programme at the Department of Nursing \& Midwifery at the University of Highlands and Islands. The wider PhD project is entitled "Technology Enabled Dementia Education and Support for Healthcare Professionals in Rural Scotland" and is directly funded by the European Social Fund and Scottish Funding Council as part of Developing Scotland's Workforce in the Scotland 2014-2020 European Structural and Investment Fund Programme. 
Availability of data and materials

Not applicable.

\section{Declarations}

Ethics approval and consent to participate

Not applicable.

\section{Consent for publication}

Not applicable.

\section{Competing interests}

The authors declare that they have no competing interests.

\section{Author details}

${ }^{1}$ Department of Nursing \& Midwifery, School of Health, Social Care \& Life Sciences, University of the Highlands and Islands, Centre for Health Science, Old Perth Road, Inverness IV2 3JH, UK. ${ }^{2}$ Learning and Teaching Academy, University of the Highlands and Islands, An Lòchran, Inverness Campus, Inverness IV2 $5 \mathrm{NA}$, UK. ${ }^{3}$ School of Health in Social Science, University of Edinburgh, Buccleuch Place, Edinburgh EH8 9LN, UK. ${ }^{4}$ Faculty of Social Sciences and Health, Durham University, Arthur Holmes Building, Lower Mountjoy, South Road, Durham DH1 3LE, UK. ${ }^{5}$ Highland Health Sciences Library, Centre for Health Science, Old Perth Road, Inverness IV2 3JH, UK.

Received: 6 October 2020 Accepted: 28 July 2021

Published online: 21 September 2021

\section{References}

1. Downs M, Turner S, Bryans M, Wilcock J, Keady J, Levin E, et al. Effectiveness of educational interventions in improving detection and management of dementia in primary care: cluster randomised controlled study. BMJ. 2006:332(7543):692-6.

2. World Health Organization. Dementia: a public health priority. 2012. https://www.who.int/mental_health/publications/dementia_report_ 2012/en/. Accessed 10 May 2020.

3. World Health Organization. Dementia. 2020. https://www.who.int/newsroom/fact-sheets/detail/dementia. Accessed 18 June 2020.

4. Alzheimer's Research UK. Dementia Statistics Hub: number of people in the UK. 2018. https://www.dementiastatistics.org/statistics/numbers-ofpeople-in-the-uk/. Accessed 18 May 2020.

5. Public Health Scotland. Dementia. 2019. http://www.healthscotland.scot/ health-topics/dementia. Accessed 22 May 2020.

6. Scottish Government. Scotland's national dementia strategy 2010. 2010 https://www2.gov.scot/Publications/2010/09/10151751/11. Accessed 26 May 2020.

7. Scottish Government. Scotland's national dementia strategy 2013-2016. 2013. https://www2.gov.scot/Topics/Health/Services/Mental-Health/ Dementia/DementiaStrategy1316. Accessed 26 May 2020.

8. Scottish Government. National dementia strategy: 2017-2020. 2017. https://www.gov.scot/publications/scotlands-national-dementia-strat egy-2017-2020/pages/3/. Accessed 26 May 2020

9. Surr C, Baillie L, Waugh A, Brown M. Position Paper: The importance of including dementia in pre and post-qualifying curricula for health and social care professionals. 2017. https://www.dementiauk.org/wp-conte nt/uploads/2017/11/HEDN-Position-Paper-for-Professional-Bodies-Colle ges-Nov-2017.pdf. Accessed 15 May 2020.

10. Cowdell F. The care of older people with dementia in acute hospitals. Int J Older People Nurs. 2010;5(2):83-92.

11. Elliott K, Scott J, Stirling C, Martin A, Robinson A. Building capacity and resilience in the dementia care workforce: a systematic review of interventions targeting worker and organizational outcomes. Int Psychogeriatr. 2012;24(6):882-94.

12. Nolan L. Caring for people with dementia in the acute setting: a study of nurses' views. Br J Nurs. 2007;16(7):419-22.

13. Timmons S, O'Shea E, O'Neill D, Gallagher P, de Siun A, McArdle D, et al. Acute hospital dementia care: results from a national audit. BMC Geriatr. 2016;16:113.
14. Bentley M, Kerr R, Ginger M, Karagoz J. Behavioural change in primary care professionals undertaking online education in dementia care in general practice. Aust J Prim Health. 2019;25(3):244-9.

15. Edwards R, Voss S, Iliffe S. The development and evaluation of an educational intervention for primary care promoting person-centred responses to dementia. Dementia. 2015;14(4):468-82.

16. Sass C, Burnley N, Drury M, Oyebode J, Surr C. Factors associated with successful dementia education for practitioners in primary care: an indepth case study. BMC Med Educ. 2019;19:393.

17. Irvine A, Bourgeois M, Billow M, Seeley J. Internet training for nurse aides to prevent resident aggression. J Am Med Dir Assoc. 2007;8(8):519-26.

18. Tullo E, Allan L. What should we be teaching medical students about dementia? Int Psychogeriatr. 2011;23(7):1044-50.

19. Kirkwood A, Price L. Technology-enabled learning implementation: handbook. Version 1. Canada: Commonwealth of Learning; 2016.

20. Anohina A. Analysis of the terminology used in the field of virtual learning. Educ Technol Soc. 2005;8(3):91-102.

21. Gordon N. Flexible Pedagogies: technology-enhanced learning. 2014 https://www.advance-he.ac.uk/knowledge-hub/flexible-pedagogiestechnology-enhanced-learning. Accessed 19 Mar 2021.

22. Vaona A, Banzi R, Kwag K, Rigon G, Cereda D, Pecoraro V, et al. E-learning for health professionals. Cochrane Database Syst Rev. 2018;1(1):CD011736.

23. George P, Papachristou N, Belisario J, Wang W, Wark P, Cotic Z, et al. Online eLearning for undergraduates in health professions: a systematic review of the impact on knowledge, skills, attitudes and satisfaction. J Glob Health. 2014;4(1):010406.

24. Scerri A, Innes A, Scerri C. Dementia training programmes for staff working in general hospital settings - a systematic review of the literature. Aging Ment Health. 2017;21(8):783-96.

25. Surr C, Gates $C$. What works in delivering dementia education or training to hospital staff? A critical synthesis of the evidence. Int J Nurs Stud. 2017;75:172-88.

26. Horner B, Watson N, Hill A, Etherton-Beer C. Description, and pilot evaluation, of novel staff education to improve care of confused older inpatients. Aust J Adv Nurs. 2014;31(2):5-12.

27. Surr C, Gates C, Irving D, Oyebode J, Smith S, Parveen S, et al. Effective dementia education and training for the health and social care workforce: a systematic review of the literature. Rev Educ Res. 2017;87(5):966-1002.

28. Scerbe A, O'Connell M, Astell A, Morgan D, Kosteniuk J, DesRoches A. Digital tools for delivery of dementia education for health-care providers: a systematic review. Educ Gerontol. 2019;45(11):681-99.

29. Allen M. What was Web 2.0? Versions as the dominant mode of internet history. New Media Soc. 2013;15(2):260-75.

30. Greenhow C, Robelia B, Hughes J. Learning, teaching, and scholarship in a digital age: Web 2.0 and classroom research: what path should we take now? Educ Res. 2009;38(4):246-59.

31. Ofcom. Connected Nations 2020 Scotland report. 2020. https://www. ofcom.org.uk/_data/assets/pdf_file/0021/209442/connected-nations2020-scotland.pdf. Accessed 19 Mar 2021.

32. Cook D, Beckman T, Bordage G. Quality of reporting of experimental studies in medical education: a systematic review. Med Educ. 2007;41(8):737-45.

33. Kirkpatrick D. Great ideas revisited. Techniques for evaluating training programs. Revisiting Kirkpatrick's four-level model. Train Dev. 1996;50(1):54-9.

34. Moher D, Liberati A, Tetzlaff J, Altman D, The PRISMA Group. Preferred reporting items for systematic reviews and meta-analyses: the PRISMA statement. PLoS Med. 2009;6(7):e1000097.

35. Muirhead K, Macaden L, Clarke C, Smyth K, Polson R, O'Malley C. The characteristics of effective technology-enabled dementia education for health and social care practitioners: protocol for a mixed studies systematic review. Syst Rev. 2019;8(1):316.

36. Cochrane Effective Practice and Organisation of Care (EPOC). EPOC resources for review authors. 2017. https://epoc.cochrane.org/resou rces/epoc-resources-review-authors. Accessed 18 June 2021.

37. Drummond K, Murphy-Reyes A. Chapter 6: Quantitative research designs: experimental, quasi-experimental, and descriptive. In: Nutrition research: concepts and applications. Burlington: Jones \& Bartlett Learning; 2018. 
38. Shadish W, Cook T, Campbell D. Experimental and quasi-experimental designs for generalized causal inference. Boston: Houghton Mifflin Company; 2002.

39. Gopalan M, Rosinger K, Ahn J. Use of quasi-experimental research designs in education research: growth, promise, and challenges. Rev Res Educ. 2020;44(1):218-43.

40. Wludyka P. Study designs and their outcomes. In: Macha K, McDonough J, editors. Epidemiology for advanced nursing practice. Sudbury: Jones \& Bartlett Learning; 2012.

41. Harris A, McGregor J, Perencevich E, Furuno J, Zhu J, Peterson D, et al. The use and interpretation of quasi-experimental studies in medical informatics. J Am Med Inform Assoc. 2006;13(1):16-23.

42. Seo D, Lee J. Web_2.0 and five years since: how the combination of technological and organizational initiatives influences an organization's long-term Web_2.0 performance. Telemat Inform. 2016;33(1):232-46.

43. Reio T, Rocco T, Smith D, Chang E. A critique of Kirkpatrick's evaluation model. New Horiz Adult Educ Hum Resour Dev. 2017;29(2):35-53.

44. Guerci M, Bartezzaghi E, Solari L. Training evaluation in Italian corporate universities: a stakeholder-based analysis. Int J Train Dev. 2010;14(4):291-308.

45. Hammick M, Dornan T, Steinert Y. Conducting a best evidence systematic review. Part 1: from idea to data coding BEME guide no. 13. Med Teach. 2010;32(1):3-15.

46. Hong Q, Pluye P, Fàbregues S, Bartlett G, Boardman F, Cargo M et al. Mixed Methods Appraisal Tool (MMAT), version 2018. 2018. http:// mixedmethodsappraisaltoolpublic.pbworks.com/w/file/fetch/12791 6259/MMAT_2018_criteria-manual_2018-08-01_ENG.pdf. Accessed 3 Mar 2020.

47. Reed D, Cook D, Beckman T, Levine R, Kern D, Wright S. Association between funding and quality of published medical education research. JAMA. 2007;298(9):1002-9.

48. Hong Q, Fàbregues S, Bartlett G, Boardman F, Cargo M, Dagenais P, et al. The Mixed Methods Appraisal Tool (MMAT) version 2018 for information professionals and researchers. Educ Inf. 2018;34(4):1-7.

49. Reed D, Beckman T, Wright S, Levine R, Kern D, Cook D. Predictive validity evidence for medical education research study quality instrument scores: quality of submissions to JGIM's Medical Education Special Issue. J Gen Intern Med. 2008;23(7):903-7.

50. Cook D, Reed D. Appraising the quality of medical education research methods: the medical education research study quality instrument and the Newcastle Ottawa Scale-Education. Acad Med. 2015;90(8):1067-76.

51. Page M, McKenzie J, Bossuyt P, Boutron I, Hoffmann T, Mulrow C, et al. The PRISMA 2020 statement: an updated guideline for reporting systematic reviews. BMJ. 2021;372:n71.

52. Chao H, Kaas M, Su Y, Lin M, Huang M, Wang J. Effects of the advanced innovative Internet-based communication education program on promoting communication between nurses and patients with dementia. J Nur Res. 2016;24(2):163-72.

53. Cobbett S, Redmond S, LeBlanc A, MacNaughton-Doucet L, Edgecombe $\mathrm{N}$, Helpard H. On-line dementia education: cultivating nursing students' comprehension, application and critical thinking skills. Perspectives 2016:39(1):7-14.

54. De Witt JB, Brazil K, Passmore P, Buchanan H, Maxwell D, Mcllfatrick S, et al. Evaluation of the impact of telementoring using $\mathrm{ECHO} \odot$ technology on healthcare professionals' knowledge and self-efficacy in assessing and managing pain for people with advanced dementia nearing the end of life. BMC Health Serv Res. 2018;18(1):228.

55. Helms A, Denson K, Brown D, Simpson D. One specialty at a time: achieving competency in geriatrics through an e-learning neurology clerkship module. Acad Med. 2009;84(Supplement):S67-9.

56. Hobday J, Gaugler J, Mittelman M. Feasibility and utility of online dementia care training for hospital staff: the CARES dementia-friendly hospital ${ }^{\mathrm{TM}}$ program. Res Gerontol Nurs. 2017;10(2):58-65.

57. Hobday J, Savik K, Gaugler J. An internet-based multimedia education prototype to enhance late-stage dementia care: formative research results. Geriatr Nurs. 2010;31(6):402-11.

58. Hobday J, Savik K, Smith S, Gaugler J. Feasibility of internet training for care staff of residents with dementia: the CARES program. J Gerontol Nurs. 2010;36(4):13-21.
59. Irvine A, Beaty J, Seeley J, Bourgeois M. Use of a dementia training designed for nurse aides to train other staff. J Appl Gerontol. 2013;32(8):936-51.

60. Jones C, Moyle W. Sexuality \& dementia: an eLearning resource to improve knowledge and attitudes of aged-care staff. Educ Gerontol. 2016;42(8):563-71.

61. Kimzey M, Mastel-Smith B, Alfred D. The impact of educational experiences on nursing students' knowledge and attitudes toward people with Alzheimer's disease: a mixed method study. Nurse Educ Today. 2016:46:57-63.

62. Luconi F. Exploring rural family physicians'learning from a web-based continuing medical education program on Alzheimer's disease: a pilot study. Ph.D. thesis, McGill University; 2008. https://www.learntechlib. org/p/126856/. Accessed 8 Feb 2020.

63. Matsumura Y, Shinno H, Mori T, Nakamura Y. Simulating clinical psychiatry for medical students: a comprehensive clinic simulator with virtual patients and an electronic medical record system. Acad Psychiatry. 2018;42(5):613-21.

64. Rababa M, Masha'al D. Using branching path simulations in critical thinking of pain management among nursing students: experimental study. Nurse Educ Today. 2020;86:104323.

65. Ruiz J, Smith M, van Zuilen M, Williams C, Mintzer M. The educational impact of a computer-based training tutorial on dementia in long term care for licensed practice nursing students. Gerontol Geriatr Educ. 2006;26(3):67-79.

66. Tomaz J, Mamede S, Filho J, Roriz Filho J, van der Molen H. Effectiveness of an online problem-based learning curriculum for training family medical doctors in Brazil. Education for Health. 2015;28(3):187-93.

67. Tsai P, Kitch S, Beck C, Jakobs T, Rettiganti M, Jordan K, et al. Using an interactive video simulator to improve certified nursing assistants' dressing assistance and nursing home residents' dressing performance: a pilot study. Comput Inform Nurs. 2018;36(4):183-92.

68. Vollmar H, Mayer H, Ostermann T, Butzlaff M, Sandars J, Wilm S, et al. Knowledge transfer for the management of dementia: a cluster-randomised trial of blended learning in general practice. Implement Sci. 2010;5:1.

69. Westmoreland G, Counsell S, Tu W, Wu J, Litzelman D. Web-based training in geriatrics for medical residents: a randomized controlled trial using standardized patients to assess outcomes. J Am Geriatr Soc. 2010;58(6):1163-9.

70. Bandura A. Self-efficacy mechanism in human agency. Am Psychol. 1982;37(2):122-47.

71. Fishbein M, Ajzen I. Predicting and changing behavior: the reasoned action approach. 1st ed. New York: Psychology Press (Taylor and Francis Group); 2010.

72. Cook D, Levinson A, Garside S, Dupras D, Erwin P, Montori V. Internetbased learning in the health professions: a meta-analysis. JAMA. 2008;300(10):1181-96.

73. Travers C, Beattie E, Martin-Khan M, Fielding E. A survey of the Queensland healthcare workforce: attitudes towards dementia care and training. BMC Geriatr. 2013;13(1):101

74. Ertmer P, Ottenbreit-Leftwich A. Removing obstacles to the pedagogical changes required by Jonassen's vision of authentic technology-enabled learning. Comput Educ. 2013;64(1):175-82.

75. Chater K, Hughes N. Strategies to deliver dementia training and education in the acute hospital setting. J Res Nurs. 2013;18(6):578-93.

76. Serhan D. Transitioning from face-to-face to remote learning: students' attitudes and perceptions of using Zoom during COVID-19 pandemic. Int J Technol Educ Sci. 2020;4(4):335-42.

77. Cook D, Levinson A, Garside S. Time and learning efficiency in Internetbased learning: a systematic review and meta-analysis. Adv Health Sci Educ. 2010;15(5):755-70.

78. Harris J, Felix L, Miners A, Murray E, Michie S, Ferguson E, et al. Adaptive e-learning to improve dietary behaviour: a systematic review and costeffectiveness analysis. Health Technol Assess. 2011;15(37):1-160.

79. Clark E, Draper J, Taylor R. Healthcare education research: the case for rethinking hierarchies of evidence. J Adv Nurs. 2018;74(11):2480-3.

80. McCabe M, Davison T, George K. Effectiveness of staff training programs for behavioral problems among older people with dementia. Aging Ment Health. 2007;11(5):505-19. 
81. Perry M, Drašković I, Lucassen $P$, Vernooij-Dassen $M$, van Achterberg T, Rikkert M. Effects of educational interventions on primary dementia care: a systematic review. Int J Geriatr Psychiatry. 2011;26(1):1-11.

82. Norman G. RCT = results confounded and trivial: the perils of grand educational experiments. Med Educ. 2003;37(7):582-4.

83. Sullivan G. A primer on the validity of assessment instruments. J Grad Med Educ. 2011;3(2):119-20.

84. Unwin L, Felstead A, Fuller A, Bishop D, Lee T, Jewson N, et al. Looking inside the Russian doll: the interconnections between context, learning and pedagogy in the workplace. Pedagog Cult Soc. 2007;15(3):333-48.

85. Rogers P. Using programme theory to evaluate complicated and complex aspects of interventions. Evaluation. 2008;14(1):29-48.
86. Li T, Higgins J, Deeks J. Chapter 5: Collecting data. In: Higgins J, Thomas J, Chandler J, Cumpston M, Li T, Page M et al, editors. Cochrane handbook for systematic reviews of interventions. Version 6.0. 2019. https://training. cochrane.org/handbook/current/chapter-05. Accessed 4 Apr 2020.

87. Kirkpatrick D, Kirkpatrick J. Evaluating training programs: the four levels. 3rd ed. California: Berrett-Koehler Publishers; 2006.

\section{Publisher's Note}

Springer Nature remains neutral with regard to jurisdictional claims in published maps and institutional affiliations.
Ready to submit your research? Choose BMC and benefit from:

- fast, convenient online submission

- thorough peer review by experienced researchers in your field

- rapid publication on acceptance

- support for research data, including large and complex data types

- gold Open Access which fosters wider collaboration and increased citations

- maximum visibility for your research: over 100M website views per year

At BMC, research is always in progress.

Learn more biomedcentral.com/submissions 